
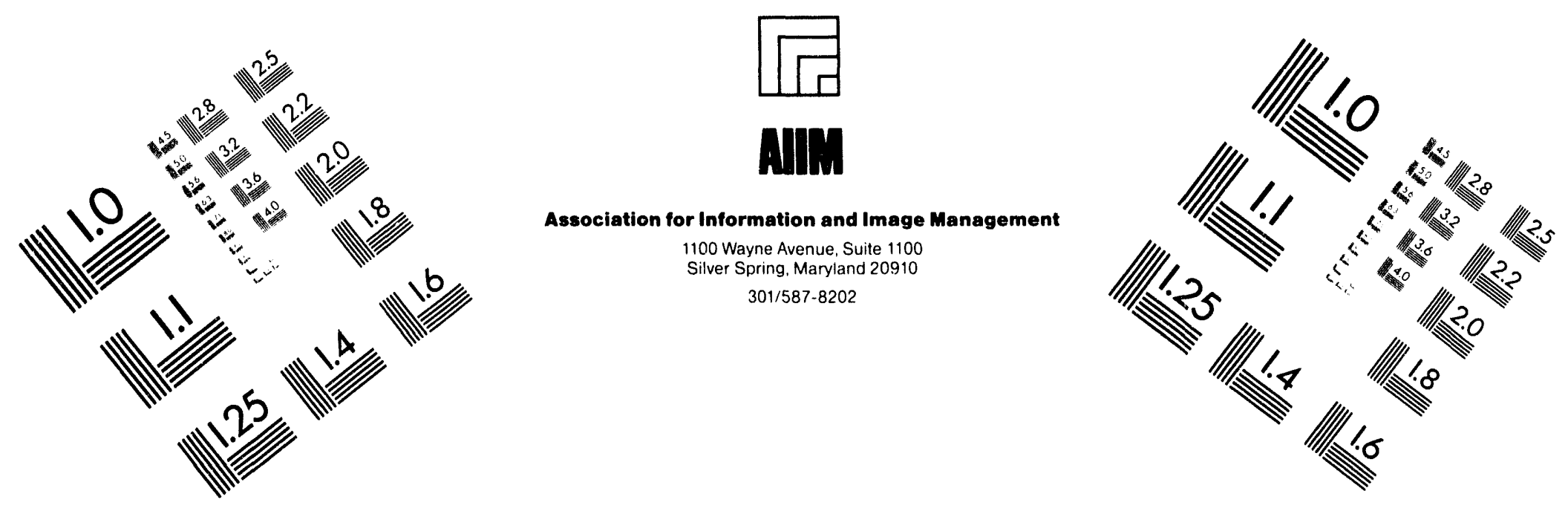

Centimeter

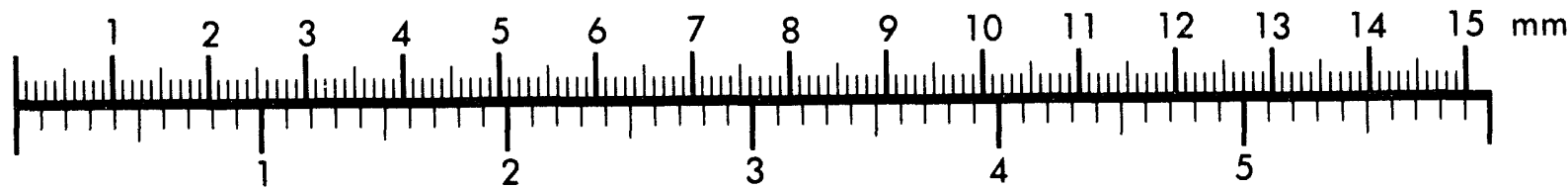

Inches
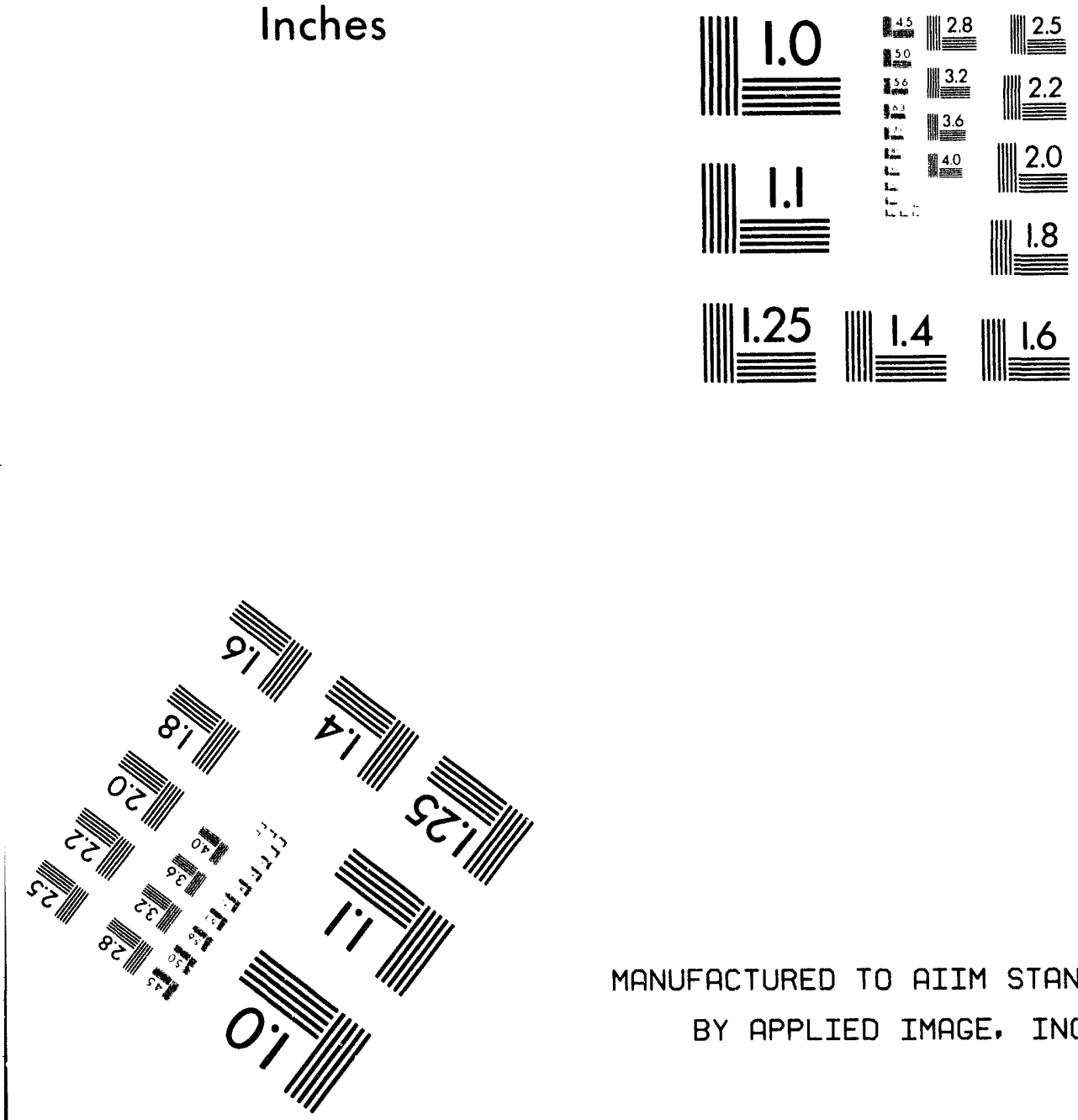

MANUFACTURED TO AIIM STANDARDS

BY APPLIED IMAGE. INC.

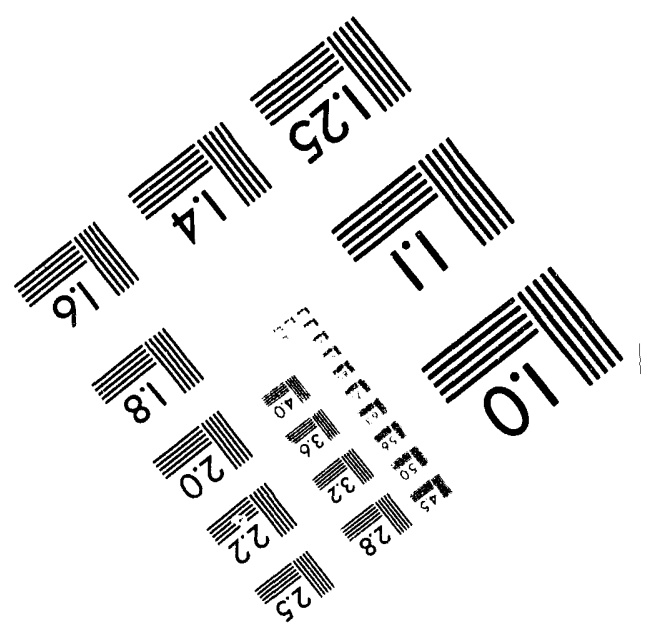



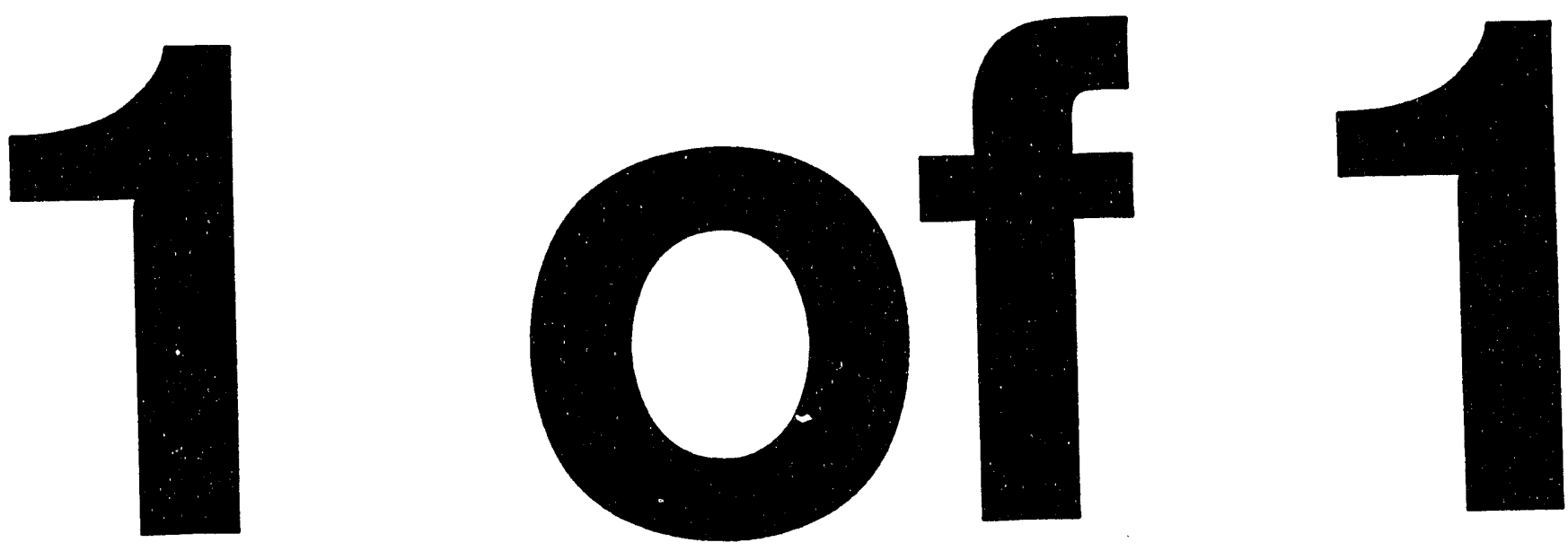
Note: This is a preprint of a paper being submitted for publication. Contents of this paper should not be quoted nor referred to without permission of the author(s).

Submitted to the NATO Conference

Elounda (Crete), Greece

September 6-17, 1993

\title{
TIME-RESOLVED DIAGNOSTICS OF EXCIMER LASER-GENERATED ABLATION PLASMAS USED FOR PULSED LASER DEPOSITION
}

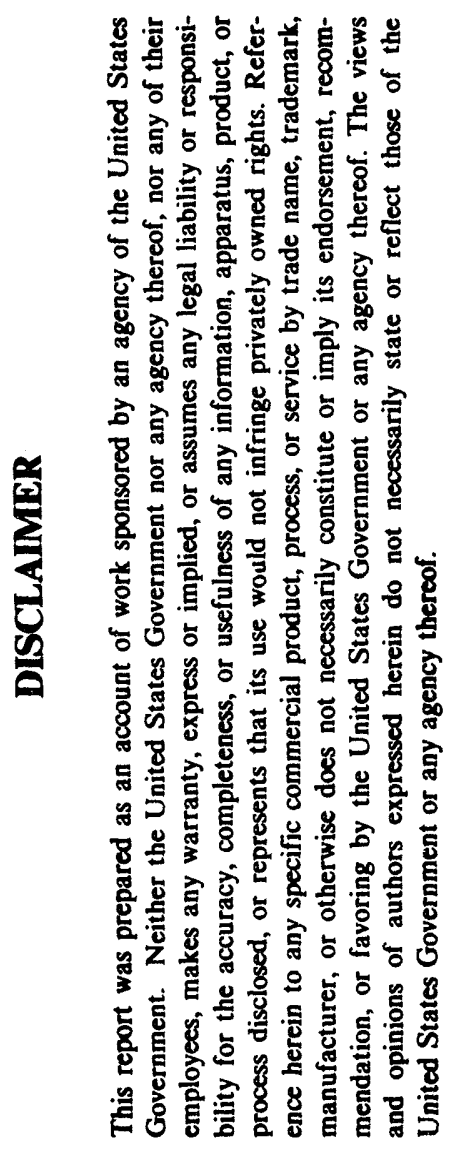

\author{
DAVID B. GEOHEGAN
}

\begin{abstract}
"The submitted manuscript has been authored by a contractor of the U.S. Government under contract No. DE-AC05$84 O R 21400$. Accordingly, the U.S. Government retains a U.S. Government retains a to publish or reproduce the published form of this contribution, or allow others to do contribution, or allow others to do purposes."
\end{abstract}

Solid State Division Oak Ridge National Laboratory P. O. Box 2008

Oak Ridge, Terınessee 37831-6056

managed by

MARTIN MARIETTA ENERGY SYSTEMS, INC. for the

U.S. DEPARTMENT OF ENERGY under contract DE-AC05-84OR21400 


\title{
TIME-RESOLVED DIAGNOSTICS OF EXCIMER LASER-GENERATED ABLATION PLASMAS USED FOR PULSED LASER DEPOSITION
}

\author{
DAVID B. GEOHEGAN \\ Oak Ridge National Laboratory \\ Solid State Division, MS 6056 \\ P.O. Box 2008 \\ Oak Ridge, Tennessee 37831-6056 \\ USA
}

\begin{abstract}
Characteristics of laser plasmas used for pulsed laser deposition (PLD) of thin films are examined with four in situ diagnostic techniques: optical emission spectroscopy, optical absorption spectroscopy, ion probe studies, and gated ICCD (intensified chargecoupled-device array) fast photography. These four techniques are complementary and permit simultaneous views of the transport of ions, excited states, ground state neutrals and ions, and hot particulates following $\mathrm{KrF}$ laser ablation of $\mathrm{YBCO}, \mathrm{BN}$, graphite and $\mathrm{Si}$ in vacuum and background gases. The implementation and advantages of the four techniques are first described in order to introduce the key features of laser plasmas for pulsed laser deposition. Aspects of the interaction of the ablation plume with background gases (i.e., thermalization, attenuation, shock formation) and the collision of the plasma plume with the substrate heater are then summarized. The techniques of fast ICCD photography and gated photon counting are then applied to investigate the temperature, velocity, and spatial distribution of hot particles generated during $\mathrm{KrF}$ ablation of $\mathrm{YBCO}, \mathrm{BN}, \mathrm{Si}$ and graphite. Finally, key features of fast imaging of the laser ablation of graphite into high pressure rare gases are presented in order to elucidate internal reflected shocks within the plume, redeposition of material on a surface, and formation of hot nanoparticles within the plume.
\end{abstract}

\section{Introduction}

Since the first successful application of pulsed laser deposition (PLD) to the growth of the high- $T_{c}$ superconductor materials in 1987,1 PLD has quickly matured into a versatile technique for film growth of many novel materials. ${ }^{2-7}$ Much of the current understanding of the complex physics involved in PLD was learned from earlier work in the field ${ }^{8}$ and from work at much higher laser energy densities, such as those used for laser fusion. ${ }^{9-12}$ Because many applications now involve laser ablation, a concerted effort to characterize the ablation process at the laser intensities used for PLD is underway and fast, in situ diagnostics are being developed to efficiently correlate gas-phase plume conditions with film properties. ${ }^{3,4,6,7}$

The details of the physical phenomena associated with (1) the laser-target interaction, (2) the characteristics and dynamics of the subsequent laser plasma, and (3) the deposition process, during PLD are still being revealed by a wide variety of diagnostic techniques. ${ }^{13}$ In this paper, examples of the capabilities of four in situ diagnostic techniques (optical emission spectroscopy, optical absorption spectroscopy, ion probe studies, and gated ICCD fast photography) are presented in order to summarize some of the characteristics of laser plasmas used for pulsed laser deposition. Far from presenting a complete picture, this paper hopes to serve as an introduction to many key outstanding questions regarding the PLD process.

It is essential to note that no single diagnostic technique presents an adequate characterization of the PLD process. Ideally, several techniques should be implemented 
simultaneously to give an overall picture of the process parameters. In addition, the techniques should be applicable in situ, i.e. in the PLD chamber, as process monitors.

Figure 1: Block diagram of experimental apparatus useful for temporally (5 ns) and spatiallyresolved optical emission and absorption spectroscopy, ion probe measurements, and fast photography of laser plumes used for PLD.

\section{Results and Discussion}

\subsection{EXPERIMENTAL APPARATUS}

The experimental configuration for spatially-and temporally-resolved optical emission spectroscopy, optical absorption spectroscopy, ion probe measurements and fast ICCD-photography is diagrammed in Fig. 1, and has been described before. ${ }^{13-23}$ Only a few essential details will be given here.

A 1-meter spectrometer (resolution $0.04 \AA$ ) is desirable in order to observe the spectral broadening of atomic lines in the laser plasma. A pulsed Xe flashlamp provides a structured continuum which is collimated and focused to $0.5 \mathrm{~mm}$ beam width through the plume for optical absorption measurements on strong resonance lines of atoms and ions in the plume. 15,16,21,23 These lines are Stark broadened in the laser plasma to widths greater than the resolution of the spectrometer, permitting such a simple polychromatic source to be used. 


\subsection{OPTICAL ABSORPTION AND EMISSION SPECTROSCOPY}

An example of the utility of this technique is given in Fig. 2, where the $\mathrm{Y}^{2} \mathrm{~F}_{7 / 2}-{ }^{2} \mathrm{D}_{5 / 2}$ transition at $4102.38 \AA$ is investigated in (a) absorption and (b) emission at a distance of $2 \mathrm{~mm}$ from the surface of a $\mathrm{YBCO}$ (i.e., $\mathrm{Y}_{1} \mathrm{Ba}_{2} \mathrm{Cu}_{3} \mathrm{O}_{7-x}$ ) pellet, $400 \mathrm{~ns}$ following irradiation by $1 \mathrm{~J} \mathrm{~cm}^{-2}$ ArF-laser pulses in vacuum. This broadening (above Doppler broadening) persists to several centimeters from the pellet.

Figure 2: Spectrally broadened profiles of the $\mathrm{Y}^{2} \mathrm{~F}_{7 / 2}-{ }^{2} \mathrm{D}_{5 / 2}$ transition at $4102.38 \AA$ in (a) absorption and (b) emission at a distance of $2 \mathrm{~mm}$ from the surface of a YBCO pellet in vacuum, $400 \mathrm{~ns}$ following irradiation by $1 \mathrm{~J} \mathrm{~cm}^{-2}$ ArF-laser pulses. The lamp spectrum is included for comparison with the absorption profile. 
Varying the observation time after positioning the spectrometer wavelength to the center of the absorption and emission profiles allows the temporal history of the ground state species to be compared with that of the excited state of the same species at a given distance. An example is shown in Fig. 3 for atomic barium at a distance of $5 \mathrm{~mm}$ from a YBCO pellet in vacuum. The ground state $\mathrm{Ba}$ density (from the absorption measurements) displays a low-velocity component to the plume transport not revealed by the $\mathrm{Ba}^{*}$ plume fluorescence. Since the lifetimes of the excited states are known to be $\sim 10 \mathrm{~ns}$, emission from these excited states after several microseconds must be the result of collisions in the plume. As the density of the plume decreases, interplume collisions become less frequent and the emission ceases. However, a large population of ground state species still exists at later times.

Figure 3: (a) Temporal history for atomic $\mathrm{Ba}, 5 \mathrm{~mm}$ from the irradiated pellet as monitored by $\mathrm{Ba}^{*}$ emission and ground-state $\mathrm{Ba}$ absorption (of the xenon lamp beam) on the same $6 \mathrm{p}$ ${ }^{1} \mathrm{P}_{1}-6 \mathrm{~s}^{2}{ }^{1} \mathrm{~S}_{0}$ transition at $5535.48 \AA$. (b) The resulting TOF velocity distribution of ground state $\mathrm{Ba}$ (from the absorbance profile) shows a pronounced low velocity component not revealed by the plume fluorescence.

As an alternative to the xenon flashlamp, spectrally-narrow, $\mathrm{CW}$ hollow-cathode lamps can be used in conjunction with a photomultiplier/interference filter system to capture the entire temporal history of a desired species on a single laser shot. However, these sources are not tunable and are generally weak in intensity. Ideally, a narrow-linewidth tunable laser would be used in place of the hollow cathode lamp.

Sometimes optical absorption is possible simultaneously on strong resonance lines of ground state ions and neutrals of the same species, such as for $\mathrm{Ba}$ and $\mathrm{Ba}^{+}$in Fig. 4(a). The ground state populations of the atoms and ions can then be directly compared. As the fluence on the YBCO target is raised from 1.0 to $2.7 \mathrm{~J} \mathrm{~cm}^{-2}$, the velocity distributions increase in both most-probable speed and width, with the $\mathrm{Ba}$ neutral population developing a bimodal distribution. ${ }^{15}$ The ions in the plume maintain a smooth distribution, however, and appear to lead the neutral population slightly. From the measured peak absorbances, the $\mathrm{Ba}^{+}$ions were found to outnumber the $\mathrm{Ba}$ neutrals by at least 5 to 1 .

Comparing the time-behavior of the $\mathrm{Ba}^{*}$ fluorescence with the $\mathrm{Ba}$ and $\mathrm{Ba}^{+}$absorbance profiles in Fig. 4(b) shows that the excited state emission clearly mimics the ground state ion population. The fast component of the ground state neutral population thus appears to be partially fed from recombining ions after a collision, resulting in some of the excited state fluorescence.

In general, comparisons between optical emission, optical absorption and ion probe TOF data show that the optical emission time dependence follows that of the ion population (the 
ions are accompanied by electrons in the $\sim$ neutral plasma plume). This behavior should be expected since each emission event arises from a collision in the plasma, such as recombination between electrons and ions, electron impact excitation of neutrals, etc.

The spectroscopic measurements (Stark broadened atomic lines, large ion populations observed by optical absorption, persistence of atomic emission) indicate a robust plasma as the principal characteristic of a PLD plume. Ion probes confirm the large ion densities measured by optical absorption, and easily measure the nonlinear increase in ion density and plume velocity with increasing laser fluence. Even before the appearance of a visible plume, the laser plasma is dense enough to be well-shielded from attempts at steering by external fields (penetration limited to $\sim$ Debye lengths $\lambda_{D}<25 \mu \mathrm{m}$ ). These effects are described in more detail in Reference 13.

Figure 4: (a) Time-of-flight absorbance $[\ln (\mathrm{I} / \mathrm{I})]$ profiles for ground state $\mathrm{Ba}^{+}$and ground state $\mathrm{Ba}$ at $\mathrm{d}=1.0 \mathrm{~cm}$ following 1.0 and $2.7 \mathrm{~J} \mathrm{~cm}^{-2}$ irradiation of $\mathrm{YBCO}$ at $10^{-5}$ Torr. As the fluence is increased, the plume velocity increases and the neutrals exhibit a bimodal velocity distribution. (b) A comparison between the temporal profiles of $\mathrm{Ba}^{+}$and $\mathrm{Ba}$ ground state absorption (at $455.4 \mathrm{~nm}$ and $553.5 \mathrm{~nm}$, respectively), and $\mathrm{Ba}^{*}$ fluorescence $(553.5 \mathrm{~nm}$, not to scale) shows that the neutral fluorescence follows the ground state ion population and the fast component of the ground state neutral population.

\subsection{INTERACTIONS OF ABLATION PLUMES WITH BACKGROUND GASES}

2.3.1 Overview. The interaction of an ablation plume with an ambient gas during PLD is experimentally quite important for several processing considerations. The ability to operate in atmospheres of up to several hundred millitorr of background gas affords PLD a unique processing advantage over other deposition techniques. Background gases are often required to continually provide one component in a growing film which may otherwise be lost in the film growth process, such as oxygen in the case of high- $T_{c}$ superconductor growth. Experimentally, however, the film deposition rate falls dramatically with distance and pressure and the plume appears to visibly stop after a certain distance which decreases with increasing pressure. In addition, diagnostics show that the kinetic energy of the material reaching the substrate also drops dramatically with distance and pressure. Furthermore, reactions of plume 
species with background gas atoms and with the other plume constituents are increased due to the collisions induced by the background gas.

As even small amounts of background gas are admitted to the vacuum chamber, the time dependence of the optical absorption signal develops a tail extending to several milliseconds. This is a result of the fact that optical absorption is a density-sensitive technique, and it detects ground state species which remain in the probed region after having been stopped by collisions with the background gas atoms. This renders optical absorption spectroscopy nearly useless after the arrival of the species at a given distance.

2.3.2 Ion Probe Measurements. Ion probes, however, are flux-sensitive to the ions arriving at a given point, so stopped ions are not recorded. They are thus extremely useful diagnostics under such harsh conditions. The situation for ions generated following $3.0 \mathrm{~J} \mathrm{~cm}^{-2} \mathrm{KrF}$ laser ablation of YBCO in oxygen is presented in Figure 5. Figure 5(a) shows the dropoff of the integrated ion probe current with distance in both vacuum and several pressures of oxygen. In vacuum the signal drops as $\mathrm{d}^{-1.8}$, close to the expected $\mathrm{d}^{-2}$ solid angle behavior. With background oxygen (or argon), the ions decrease as $d^{-1.8} \mathrm{e}^{-b d}$, where $b$ is an attenuation coefficient in $\mathrm{cm}^{-1}$ which is, in this case, linear with pressure. The ions thus follow a simple scattering model where the initial beam intensity $I_{0}$ arriving at a given distance, $d$, is reduced according to $l=I_{0} \mathrm{e}^{-N \sigma d}$, where $\mathrm{N}$ is the number density and $\sigma$ is the scattering cross section for ions with the background gas (which should vary with velocity) and was determined to be $\sigma=2.3 \times 10^{-16} \mathrm{~cm}^{2}$.

Figure 5: (a) Integrated positive-ion charge transmitted through oxygen backpressures of $0.01,50,100,200$, and $300 \mathrm{~m}$ Torr as measured by a fast ion probe along the normal to a YBCO pellet irradiated with $3.0 \mathrm{~J}-\mathrm{cm}^{-2} 248-\mathrm{nm}$ irradiation. Fits to the data are of the form $d^{-1.8} e^{-b d}$ where $b$ is the attenuation coefficient and $d$ is the distance form the pellet. (b) Normalized ion probe current waveforms measured at $d=5.0 \mathrm{~cm}$ along the normal to a YBCO pellet irradiated with $3.0 \mathrm{~J}-\mathrm{cm}^{-2} 248-\mathrm{nm}$ irradiation in $0.01-, 25-, 50-, 75-, 100-, 150-$, and 200-mTorr oxygen.

Figure 5(b) shows the corresponding ion probe waveforms (magnitudes have been normalized for comparison) at $d=5.0 \mathrm{~cm}$ for several oxygen pressures. The plasma arrival becomes delayed significantly and the pulse-width is broadened also. It is important to note that the velocity of the incoming material (following slowing by background gases) cannot be inferred immediately from the arrival time, i.e., $v \neq d / t$. The velocity of the leading edge or peak of the 
signal is determined from the slope of a distance-time plot, discussed below. The ion probe waveforms display a splitting of the plume into a fast component which is transmitted with little delay, and a component which has undergone pronounced slowing. As the background pressure is increased, more of the plume is delayed to longer times and a single-component pulse shape is acquired past $\sim 100$ mTorr.

2.3.3 Fast ICCD Photographic Measurements. Fast ICCD photography of the plume fluorescence permits two-dimensional investigation of this phenomena. ${ }^{22}$ Figure 6 shows a direct comparison between laser ablation of YBCO into vacuum and into 100 mTorr oxygen under typical PLD conditions. These images were recorded with an ICCD camera which converts a light image into photoelectrons at a photocathode, amplifies these electrons (gain $\sim 5 \times 10^{6}$ ) through a microchannel plate (MCP), reconverts the electrons to light on a phosphor screen and captures these images with a CCD array. A fast voltage pulse temporarily removes a reverse-bias on the MCP and serves as the shutter for the camera, giving $\sim 5 \mathrm{~ns}$ resolution.

The extreme sensitivity of the ICCD camera permits the weak fluorescence due to interbeam collisions to be observed in vacuum [Figs. $6(a)-6(f)$ ] and reveals that the highly forward-directed spatial distribution does not change significantly after Fig. 6(a). In 100 m'l orr oxygen [Figs. $6(\mathrm{~g})-6(\mathrm{l})$ ], the dense plasma expands in much the same manner during the first microsecond, virtually unhindered by the background oxygen. However, collisions on the expanding front of the plume lead to increased fluorescence there, while the emission from the rest of the plume remains unperturbed.

As the plume continues to expand, the pressure in the plume continues to decrease, the contact front is slowed more noticeably, and the shielded slower components propagate to coalesce with the slowed material on the contact front. This coalescence and slowing can be seen from $\Delta t=1.0-3.0 \mu \mathrm{s}$ in Fig. $6(\mathrm{~h})-6(\mathrm{j})$ as the emission becomes dominated by that at the expansion front. During this period, the plume emission intensity is located in two principal spatial regions reminiscent of the two distributions of ions observed with the ion probe. The plume continues to slow behind this sharp front until nearly stopped $\left(v \sim 0.06 \mathrm{~cm}-\mu \mathrm{s}^{-1}\right.$ at $\Delta t=8.0 \mu$ s under these conditions).

This formation of a stable "shock" structure is shown again in the set of ICCD photographs of Fig. 7, in which a heater is placed at $d=5 \mathrm{~cm}$ from a YBCO pellet irradiated by a $2.5 \mathrm{~J} \mathrm{~cm}^{-2}$ $\mathrm{KrF}$ laser pulse in $200 \mathrm{mT}$ Tor oxygen. The digitized intensity data has been evenly divided into five contours to reveal the fainter regions of emission. Observing the penetration of the plume by eye (or ordinary camera) would lead to the judgment that the plume had stopped short of the heater $5 \mathrm{~cm}$ away. However, increasing the exposure widths and MCP-gain during this set of photographs reveals a variety of new phenomena. As in Fig. 6, the plume expands unhindered during the first microsecond, forms a contact front which undergoes noticeable slowing, and coalesces into a stable propagating shock front. 
Figure 6: ICCD photographs of the visible plasma emission (exposure times $20 \mathrm{~ns}$ ) following $1.0 \mathrm{~J}-\mathrm{cm}^{-2} \mathrm{KrF} / \mathrm{YBCO}$ ablation into $[(\mathrm{a})-(\mathrm{f})] 1 \times 10^{-6}$ Torr and [Figs. (g)-(l)] 100 mTorr oxygen at the indicated delay times from the arrival of the laser

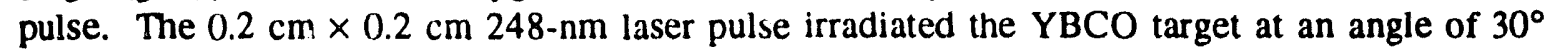
as shown. 
Figure 7: The formation and propagation of the plasma plume generated by $2.5 \mathrm{~J} \mathrm{~cm}^{-2}$ laser irradiation of YBCO in 200 mTorr oxygen as recorded by a gated, intensified CCD-array camera. Exposure times were increased from $20 \mathrm{~ns}$ to $2 \mu \mathrm{s}$. The digitized intensity data has been evenly divided into five contours to reveal the fainter regions of emission. The plume collides with a cold, stainless steel heater in $(\mathrm{h})-(\mathrm{m})$, partially rebounding to fill the space between the target and heater at $\Delta t=100 \mu \mathrm{s}$. Weak emission at the target surface is just observable in $(j)-(m)$ and is due to blackbody emission from slow-moving, hot particulates.

The material does not stop, however, but continues to slowly move toward the heater. The velocity of the plume boundary just before impact with the heater (from an $R-t$ plot for the data discussed below) is $0.07 \mathrm{~cm} \mathrm{\mu s}^{-1}$, which yields a kinetic energy (for a $100 \mathrm{amu}$ atom) of $\sim 0.25 \mathrm{eV}$. The vapor clearly does not entirely stick to the heater (at room temperature in this case), but partially reflects from it. As the reflected vapor encounters the rest of the incoming plume, the collision rate and fluorescence increase to reveal the nearly thermalized layer of material at the surface. ${ }^{20,24}$ This layer appears to collectively move away from the heater while diffusing in an apparent afterglow, eventually filling the gap between target and heater as it continues to emit light for over $100 \mu \mathrm{s}$. As shown in these photos, substrates on the heater surface are bathed in an excited stagnation layer for much longer than previously realized.

2.3.4 Propagation models of plume propagation in a background gas. The propagation of the plasma plume through a background gas can be represented by several models. Two of these, the "shock" and "drag" models will be briefly discussed with regard to Fig. 8 which is a distance-time $(R-t)$ plot of the leading edge of the visible plasma emission from the ICCD images of Figure 7.

2.3.4.1 Blast wave or 'shock' models. A blast wave model has been used to describe the luminous shock front caused by the expansion of laser ablation plasmas at high background pressures. This model was developed to describe the propagation of a shock wave through a 
background gas (density $\rho_{0}$ ) caused by a the sudden release of energy, $E_{O}$, in an explosion. 25 The propagation of shock fronts through background gases follow the distance-time relations:

Blast wave or "shock" models

Spherical shock front

$$
R(t)=\xi_{0}\left(E_{\delta} / \rho_{0}\right)^{1 / 5} t^{2 / 5}
$$

Planar shock front

$$
R(t)=\xi_{0}\left(E_{\delta} \rho_{0}\right)^{1 / 3} t^{2 / 3}
$$

where $\xi_{0}$ is a constant. These models strictly apply when the mass of the ejected products is small compared with the mass of the background gas set in motion by the shock. ${ }^{25}$ a situation which does not typically apply for PL.D plumes. Furthermore, the model is designed to follow the movement of the background gas set in motion by the explosion products, while for PLD it is the motion of the target ejecta that is of interest. Nevertheless, the appearance of the sharp contact surface during plume propagation is highly suggestive of a shock wave.

As shown in Fig. 8, shock model tits to $R-t$ plots at low background pressures consistently predict greater propagation distances than actually measured at early times. Generalized fits of the form $R=a^{n}{ }^{n}$ suggest exponents $n>0.4$ for limited improvement at early times $(n=0.57$ in Fig. 8). 16,21 However, ignorirg the temporal region prior to the observed formation of the shock front, the blast wave model describes this phase of the expansion increasingly well at higher background pressures. ${ }^{26.28}$ Fits of the blast wave model to the data of Fig. 8 for times $>4 \mu$ sive excellent agreement.

Figure 8: Distance-time $(R-t)$ plots of the plume propagation along the normal to a YBCO pellet following $\mathrm{KrF}$ laser irradiation with $2.5 \mathrm{~J} \mathrm{~cm}^{-2}$ in $200 \mathrm{mTorr}$ oxygen gas as measured from ICCD images of the visible plasma emission. (a) Shock model fits to the data using $\mathrm{R}=\mathrm{at}^{\mathrm{n}}$ with $\mathrm{n}=0.4$ (single parameter fit) and $\mathrm{R}=\mathrm{at}{ }^{\mathrm{n}}$ where $\mathrm{n}$ is allowed to vary. (b) Fits to the data using two viscous drag models (i) $a=-\beta v$, predicting $R=x_{f}\left(1-e^{-\beta t}\right)$ stopping of the plume at $x_{\mathrm{f}}=5.0 \mathrm{~cm}$ and (ii) $\mathrm{a}=-\alpha \mathrm{v}^{2}$ deceleration, predicting $\mathrm{R}=\alpha^{-1} \ln \left(\alpha \mathrm{v}_{\mathrm{o}} \mathrm{t}+1\right)$ slowing of the plume. 
Piezoelectric transducer measurements of the transient pressure generated by the YBCO ablation products (under similar experimental conditions) have been shown by Dyer, et.al. to be $\sim 400$ atm. during the laser pulse. 29 This large pressure gradient causes the high density bubble to rapidly expand through the low-pressure background gas. Dyer, et. al. have shown that the low pressure $R-t$ behavior conforms to blast wave propagation after the bubble expands to a distance at which the mass of gas (density $\rho_{0}$ ) displaced by the shock wave is much greater than the ablated mass $\mathrm{M}_{0} \cdot{ }^{30}$

2.3.4.2 Viscous or 'drag' models. In order to include the early time behavior of the plume expansion, a classical drag force model can be invoked. The ejected pulse of ablation products is regarded as an ensemble which is launched with initial velocity $v_{0}$ into a medium where it experiences a viscous force proportional to its velocity (or more generally, proportional to $\mathrm{v}^{n}$ ). The equations of motion for $n=1,2$ are given below:

Viscous or "drag" models

$$
a=-\alpha v^{n}
$$

$$
\begin{aligned}
& n=1 \\
& a=-\beta v \quad[\beta] \sim s^{-1} \\
& v=v_{0} e^{-\beta t} \\
& x=\frac{v_{0}}{\beta}\left(1-e^{-\beta t}\right)
\end{aligned}
$$

$$
\begin{aligned}
& n=2 \\
& a=-\alpha v^{2} \quad[\alpha] \sim m^{-1} \\
& v=\frac{v_{0}}{1+\alpha v_{0} t} \\
& x=\frac{1}{\alpha} \ln \left(\alpha v_{0} t+1\right)
\end{aligned}
$$

Fits of the drag models to the data of Fig. 7 are given in Fig. 8(b). Both models fit the early time behavior of the expansion, and, in fact, the entire range. The $n=1$ drag model predicts that the plume will come to a halt at a distance $x_{f}=v_{0} / \beta \quad[5.0 \mathrm{~cm}$ in Fig. $8(\mathrm{~b})]$ while the $n=2$ drag model correctly predicts that the plume continues to propagate to longer distances.

However, fitting the entire shape of the ion probe waveform (for times after the formation of the visible "shock" front) with drag and shock models revealed that a generalized shock model $R=a t^{0.57}$ described the data much better than the drag model. ${ }^{21,16}$

Thus, a combination of diagnostics reveals that PLD plumes travel a path which can be described by a composite of these drag and shock models, the overlap occurring during the transition region described above when the viscous slowing of the leading edge of the plume coalesces with the trailing material to form a stable shock structure.

In summary, fast photography with ICCD sensitivity is extremely useful for studying the hydrodynamic effects of ablation plumes in background gases. As one can imagine from the photography, emission spectroscopy along a particular line-of-sight is less useful without previous knowledge of the hydrodynamics. Recall that non-emitting material is not represenied by these photographs and that absorption spectroscopy is useful principally for the leading edge of the expansion, because absorption by the trailing material is muddled with absorption from the stopped material. Ion probes, being flux sensitive, record only the flux of ions which penetrate to their positions and are therefore very useful in background gases. 


\subsection{DIAGNOSTICS OF PARTICULATE EJECTION DURING LASER ABLATION}

2.4.1 Overview. Large particles (diameters $<1 \mu \mathrm{m}$ ) are nearly always found in films grown by PLD. ${ }^{7}$ They are also a major problem in applications which attempt to utilize only the light from laser plasmas, such as for xray lithography. ${ }^{31}$ The particles have been studied by velocity filter analysis ${ }^{32}$ and laser beam deflection measurements ${ }^{33}$ which show that they travel at velocities peaked $\sim 1 \times 10^{4} \mathrm{~cm} \mathrm{~s}^{-1}$, or about 100 times slower than the velocity of the luminous plasma plume. The fraction of the mass transported to the growing film by clusters and large particulates is not known, however improved superconducting thin film properties have been obtained by separating the flux of large particles from the plasma plume $e^{32-34}$ or by vaporizing some of the particles using a second laser. ${ }^{35}$

Several theories exist regarding the particulate ejection cause and dynamics, such as subsurface heating and eruption or expulsion by shoc! waves in the target. ${ }^{36-38}$ Few in situ diagnostics have been reported to investigate particle pr.perties such as temperature, ejection time and size directly. ${ }^{33,39}$

2.4.2 Sensitive ICCD Photography of Hot Particulate Ejection During PLD. During ICCD photography of the latter stages of an expanding plasma plume, a small region of very weak emission can often be observed very near the target surface when a sufficiently long exposure is chosen. This is shown in Fig. 9 for YBCO ablation in vacuum ( $\mathrm{KrF}$ laser, $6.6 \mathrm{~J} \mathrm{~cm}^{-2}$ ) beginning at the time delay of $10 \mu \mathrm{s}$. After the strong plasma emission for $t<2 \mu \mathrm{s}$, the plume collides with a cold stainless steel heater $5 \mathrm{~cm}$ away and the dominant emission from $5 \mu \mathrm{s}<t<$ $10 \mu \mathrm{s}$ comes from enhanced collisions between incoming and rebounding material at a stagnation layer near the heater surface. In addition to the rebounding expansion front, a small glow is noted in near the pellet surface at $\Delta t=10 \mu \mathrm{s}$ in Fig. 9. At later times in vacuum, this glow clearly extends well away from the target surface and dominates remnants of the plasma emission in brightness.

Figure 9: (a) ICCD photographs tracing the appearance of glowing particulates following the expansion of the plasma plume caused by $2.5 \mathrm{~J} \mathrm{~cm}^{-2} \mathrm{KrF}$ laser ablation of YBCO in vacuum $\left(1 \times 10^{-6}\right.$ Torr). Each photograph is a different laser shot, exposures (lens $\mathrm{f}$-stops/MCP gate widths) are as follows: $2 \mu \mathrm{s}[\mathrm{f} / 32,20 \mathrm{~ns}] ; 10 \mu \mathrm{s}[\mathrm{f} / 11,200 \mathrm{~ns}] ; 100 \mu \mathrm{s}[\mathrm{f} / 4,2.2 \mu \mathrm{s}]$. 
This emission has been attributed to blackbody radiation from hot particulates ejected during the ablation process. ${ }^{20}$ Figure 10 presents a closer view of the near-target region at a YBCO pellet following $1.0 \mathrm{~J} \mathrm{~cm}^{-2} 248-\mathrm{nm}$ irradiation in vacuum at the incicated delay times. The particulates are ejected much more isotropically than the highly forward-directed plasma expansion. Although it may appear that this emission is a 'secondary' ejection process from the images of Fig. 9, the extension of the particles at $\Delta t=10 \mu \mathrm{s}$ coupled with their subsequent trajectories imply that the particles were launched at $\Delta t=0$. The velocities of these particles are approximately 100 times slower than the plasma plume, however. The individual pixels observed in the photographs are likely the result of individual photons which were detected and subsequently amplified by the MCP intensifier assembly. Detectable emission exists in this region to $\sim 3 \mathrm{~ms}$ where no pixels record counts.

Figure 10: Close up ICCD photographs of glowing particulates in the $d=0-1.5 \mathrm{~cm}$ region of a YBCO pellet following $1.0 \mathrm{~J} \mathrm{~cm}^{-2} \mathrm{KrF}$-laser irradiation, with time delays and exposures (lens $\mathrm{f} \# / \mathrm{MCP}$ gate width): $10 \mu \mathrm{s}$ (f/5.6, $200 \mathrm{~ns}), 50 \mu \mathrm{s}$ (f/4, $2 \mu \mathrm{s}), 100 \mu \mathrm{s}(\mathrm{f} / 4,2 \mu \mathrm{s}), 500 \mu \mathrm{s}(\mathrm{f} / 4$, $2 \mu s)$.

\subsubsection{Gated Photon Counting of Optical Emission Emanating from Laser Generated} Particulctes. Hot particulates are also observed for KrF-laser irradiated boron nitride $20,17,18$ and many other materials. In order to assign the nature of this long-lived emission, gated photon counting must be performed. A 0.3 -meter spectrometer with $800 \mathrm{~nm}$-blazed grating was employed with a gatable R943-02 photomultiplier to investigate the $d=1-10 \mathrm{~mm}$ spatial region near the pellet. The spectrometer was scanned in $5 \mathrm{~nm}$ intervals and photons were counted and averaged over at least 20 shots per wavelength. The raw spectra were corrected for instrumental spectral response by a calibration curve derived with a calibrated spectral lamp source. Broad emission spectra were obtained for both YBCO and BN, starting at $\sim 300 \mathrm{~nm}$ and rising into the ntar-IR such as that shown for BN between 100-150 $\mu \mathrm{s}$ in Fig. 11(a). The spectral shape was suggestive of a blackbody spectrum, and curve fits to Planck's radiation law showed good agreement [see solid curve in Fig. 11(a)]. Although both YBCO (and BN) exhibit blue target photoluminescence (and phosphorescence), the spectral shape and temporal behavior of these effects were much different. ${ }^{17}$

The emission from these hot particles can be easily identified as individual photon spikes on the photomultiplier signal (when displayed on a Tektronix 7912 oscilloscope at $\sim 100 \mu \mathrm{s}$ delay times) after the light is dispersed by a spectrometer. Discriminating and counting these photons within a scanned gate yields the time behavior of the emission. Figure 11(b) gives the time behavior of glowing particulates arriving at $d=1.1 \pm 0.1 \mathrm{~cm}$ from a BN pellet irradiated with $1.5 \mathrm{~J} \mathrm{~cm}^{-2}$ in vacuum. The brightest region of emission $(-15$ photons per microsecond 
detected at $\lambda=800 \pm 2.5 \mathrm{~nm}$ ) at this distance was found at $\Delta \mathrm{t} \sim 80 \mu \mathrm{s}$, corresponding to a velocity of $v \sim 0.013 \mathrm{~cm} \mathrm{\mu s}^{-1}$. For YBCO, the peak velocity at $1.5 \mathrm{~J} \mathrm{~cm}^{-2}$ was found to be

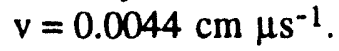

Figure 11: (a) Emission spectrum from particulates within $d=1-10 \mathrm{~mm}$ of a BN pellet, $100-150 \mu \mathrm{s}$ following $3.5 \mathrm{~J} \mathrm{~cm}^{-2} \mathrm{KrF}$ laser irradiation of $\mathrm{BN}$ in vacuum. The smooth curve is a blackbody spectrum $\rho(\lambda)=\left(8 \pi \mathrm{hc} \lambda^{-5}\right)\left(\mathrm{e}^{\mathrm{hc} / \lambda \mathrm{kT}}-1\right)^{-1}$ fit to the data corresponding to a perfect blackbody temperature of $2935 \mathrm{~K}$. The spectrum is corrected for the spectral response of the detection equipment. Error bars represent statistical counting uncertainty $( \pm 1 \sigma)$. (b) The arrival of glowing particulates at $d=1.1 \pm 0.1 \mathrm{~cm}$ following $\mathrm{KrF}$ laser $\left(1.5 \mathrm{~J} \mathrm{~cm} \mathrm{~cm}^{-2}\right)$ ablation of $B N$ in vacuum. The number of $800 \mathrm{~nm}( \pm 2.5 \mathrm{~nm})$ photons counted in a $1 \mu \mathrm{s}$ gate

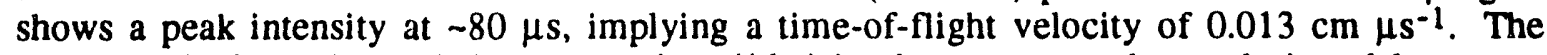
error bars indicate the statistical uncertainty $( \pm 1 \sigma)$ in photons counted at each time delay.

2.4.4 Particle Temperature Estimates from Blackbody Emission Spectra. Estimates of the temperatures of the particles were obtained for different time delays following the $\mathrm{KrF}$ laser pulse by fitting the blackbody radiation spectra [such as that in Figure 11(a)] with Planck's radiation law. The spectra shifted to longer wavelength and decreased in intensity at later times, indicating a decrease in temperature.

The derived temperatures of particulates ejected in vacuum after $3.5 \mathrm{~J} \mathrm{~cm}^{-2} \mathrm{KrF}$-laser irradiation of $\mathrm{BN}$ and $1.5 \mathrm{~J} \mathrm{~cm}^{-2}$ irradiation of YBCO are plotted in Fig. 12. Error bars indicate the temporal gate used for photon counting and the statistical uncertainty $(1 \sigma)$ in the temperatures given by the curve fits. The solid curves are fits to the data using a radiative cooling model which models the heat loss of the particles due to blackbody radiation alone. This model assumes a uniform radius, $r$, and initial temperature, $\mathrm{T}_{0}$, for the particles and the fits show that the particles cool faster than radiational cooling permits.

Figure 12 indicates that the particles are superheated above the vaporization temperature (for YBCO $\sim 2270 \mathrm{~K})^{36}$ upon ejection into vacuum. This is consistent with models predicting superheating of targets (for $\mathrm{YBCO}, 2 \mathrm{~J} \mathrm{~cm}^{-2}, \mathrm{KrF}: \mathrm{T} \sim 5200 \mathrm{~K}$ ) ${ }^{36}$ when rapid laser heating rates exceed thermal conductivity and evaporation kinetics. Superheated particles should be expected to vaporize in flight at a rate determined by the velocity of their vaporizing surface. This may contribute to the slow component to the plume transport observed by optical absorption. 
Figure 12: Particulate temperatures implied from perfect blackbody curve fits to emission spectra such as Fig. 11(a) for BN at $3.5 \mathrm{~J} \mathrm{~cm}^{-2}$ and $Y B C O$ at $1.5 \mathrm{~J} \mathrm{~cm}^{-2}$. Error bars indicate the temporal gate used for photon counting and the statistical uncertainty $(1 \sigma)$ in the temperatures given by the curve fits. The solid curves are fits to the data using a simple radiative cooling model with adjustable parameters $T_{0}$ (initial temperature) and $r$ (radius of the particles). The particles appear to be superheated above the vaporization temperature and cool faster than radiational cooling permits, presumably due to evaporation.

2.4.5 Single-Shot Trajectory and Velocity Diagnostics. By utilizing intentionally long exposures, streaks can be recorded, as shown in Fig. 13 for hot particles ejected from a $16 \mathrm{~J} \mathrm{~cm}^{-2}$ KrF-laser irradiated silicon wafer. The $200 \mathrm{~ns}$-duration exposure freezes the motion of the particles, while the second exposure ( $100 \mu \mathrm{s}$ duration) permits estimation of the particles' direction and velocity (e.g., see inset in Fig. 13). In this case, the streaked image more clearly shows the inclination of the ejected particles out of the ablation crater back toward the laser beam.

Figure 13: ICCD photographs of hot particulates ejected from a silicon wafer at a time delay of $\mathrm{Dt}=100 \mathrm{~ms}$ following $16 \mathrm{~J} \mathrm{~cm}^{-2} \mathrm{KrF}$ laser irradiation. The first exposure $(200 \mathrm{~ns}$ duration) freezes the motion of the particles, while the second exposure (100 ms duration) permits estimation of the particles' direction and velocity (e.g., see inset for isolated particle heading in the indicated direction: $v_{1}=v_{2}=3500 \mathrm{~cm} / \mathrm{s}$, from the appearance and streakedimage distances, and the prearranged delay and exposure times). 
Thus, ICCD photography and optical emission (gated photon counting) spectroscopy are extremely useful diagnostics for hot particle ejection, allowing diagnosis of the onset of ejection and estimates of particle temperature, velocity, and direction.

\subsection{ABLATION INTO HIGH-PRESSURE BACKGROUND GASES}

2.5.1 Overview. At higher gas pressures laser ablation can be utilized to produce novel clusters, such as fullerenes and doped fullerenes by the ablation of graphite into inert gases. ${ }^{40-45}$ The dynamics of fullerene assembly in the ablation plume are unclear, however production of fullerenes seems clearly related to the pressure and temperature within the confined bubble of plasma which is stopped by the high pressure ambient. Recently, the production of fullerene clusters by ablation of graphite rods into $300 \mathrm{Torr} \mathrm{He}, \mathrm{Ne}, \mathrm{Ar}$, and $\mathrm{Xe}$ has been studied by gated ICCD photography. 45,46

2.5.2 Fast ICCD Photography of Plume Expansion Dynamics and Hot Nanoparticles. The ICCD photographic investigations of the plasma plume propagation in the different gases reveal three common phases to the expansions: (1) forward motion, deceleration and stopping of the leading edge of the plume, (2) an apparent reflected shock within the plume which propagates backward and partially reflects from the graphite rod surface, leaving "redeposited" material, (3) a secondary forward propagation and coalescence of the material reflected from the rod surface, resulting in continued expansion and dissipation of the plasma and the appearance of glowing ultrafine particles. One example of these results is given here to point out several interesting effects (which are also suspected to occur in PLD plumes), including the redeposition of material on the target surface, reflected shocks within the plume, and details of the plume coalescence into a stable shock front.

A sequence of images of the visible plasma emission following laser ablation of $1 / 4^{\prime \prime}$-diameter pyrolytic graphite rods $\left(\mathrm{KrF}, 20 \mathrm{~J} \mathrm{~cm}^{-2}\right)$ into 300 Torr argon is presented in Fig. 14. The plume is extremely bright initially, and the exposures and MCP-gain were continually adjusted in acquiring the images for Fig. 14 as the plume emission decreased in intensity over ten orders of magnitude. Several reflected shocks within the hot plasma bubble are evident during the plume expansion. The shape and details of the plasma expansion were extremely reproducible from shot to shot for $\Delta \mathrm{t} \leq 100 \mu \mathrm{s}$. For $\Delta \mathrm{t}>100 \mu \mathrm{s}$, the plume often displayed a less-reproducible chaotic, turbulent behavior.

The plume propagation follows a drag model over the first microsecond. 45 Within $300 \mathrm{ns,}$ the plume is slowed dramatically and a backward-propagating front becomes noticeable at $\Delta t=$ $400 \mathrm{~ns}$. This appears to be material reflected from the front contact surface of the plume. This is more easily recognized in Fig. 15, where the corresponding images from Fig. 14 have been presented such that the digitized intensity is plotted along the vertical axis. ${ }^{46}$ At $600 \mathrm{~ns}$ this reflected component appears to reach the rod surface and from $800 \mathrm{~ns}<\Delta \mathrm{t} \leq 1 \mu \mathrm{s}$, a reflection from the rod face occurs. The portion of backward-propagating plume which extends around the side of the rod face results in plasma emission "behind" the rod surface in the images from $1 \mu \mathrm{s} \leq \Delta \mathrm{t} \leq 30 \mu \mathrm{s}$, and in a band of "redeposited" soot on the side of the rod as shown in the inset of Fig. 14.

Another reflection from the front contact surface appears to occur circa $3 \mu \mathrm{s}$, resulting in another set of interfering traveling waves within the plume and an apparent complex interference between forward- and backward-going material during $5 \mu \mathrm{s} \leq \Delta \mathrm{t} \leq 10 \mu \mathrm{s}$.

The final expansion stage is presented in Fig. 14 for the $10 \mu s \leq \Delta t \leq 100 \mu$ s images in which a stable forward-propagating front of material slowly emerges to generally mainiain its shape to very long times. The plasma emission diminishes to yield the glow from hot nanoparticulates which are separated spatially from the rod surface region, as indicated in the $500 \mu \mathrm{s}$ imagt of Fig. 14. 
Figure 14: Gated ICCD photographs of the visible plume following KrF-laser ablation (20 J cm-2, $46 \mathrm{~ns}$ FWHM pulse) of graphite into 300 Torr Argon at the indicated time delays following laser arrival. Exposures were varied and normalized (see text). The side-on view of the graphite rod, scale, and band of redeposited soot is shown in the inset (the rod surface in the $20-100 \mu \mathrm{s}$ images is offset by 1.5 scale-mm further left). Several reflected shocks are evident. The first begins $\sim 300 \mathrm{~ns}$, resulting in backward-moving material leading to redeposition of soot on the rod sides, and another reflected shock from the rod surface $(\sim 1 \mu \mathrm{s})$. This process is repeated, the plume expanding in stages, until glowing nanoparticulates are observed away from the target at very long times (see $500 \mu$ s image). 
Figure 15: Selected data from Fig. 14 tracing the evolution of the plasma plume from KrFlaser irradiated graphite into 300 Torr Argon. In this 3D representation, the intensity of the light in Fig. 14 is plotted along the vertical axis. Following rapid expansion in the first $300 \mathrm{ns,}$ a reflected shock propagates back and collides with the surface of the rod from 400 ns to $1 \mu \mathrm{s}$. Plasma emission wrapping around the side of the rod causes the apparent emission "behind" the rod surface. The reflection of material from the rod surface influences a secondary forward expansion.

2.5.3 Soot Deposition and Anclysis. Material was collected for analysis by Fourier Transform Mass Spectrometry (FTMS) from the redeposited soot on the graphite rod and from the soot deposited in the region of the glowing particulates noted in the ICCD photographs, as shown in Fig. 16. The soot which was redeposited on the graphite rod following ablation was highly fulierene deficient compared to the material collected on a sample disk $1.5 \mathrm{~cm}$ from the rod. ${ }^{45}$ The difference in fullerene content between the material deposited on the rod and on the sample disk may be rationalized if one takes into account that disk soot deposition occurs after 500-1000 $\mu$ s (the third phase) when the ablated material has cooled and mixed well with the buffer gas.

The soot deposited on the rod, however, consists of ultrafine particles which either condensed from atomic vapor durin's the redeposition process or evaporated from the rod. The backward plasma results in the deposition of this material on the rod surface before fullerene growth can take place. A fraction of the backward-moving material reflects from the rod surface and travels with the long-lived plasma, anneals, and gives rise to fullerene growth. These growing clusters finally deposit on the sample disk. 
Figure 16: ICCD photograph of glowing nanoparticulates (100 $\mu$ s exposure, starting at a time delay of $600 \mu \mathrm{s}$ ) following $20 \mathrm{~J} \mathrm{~cm}-2 \mathrm{KrF}$ laser-irradiation of pyrolytic graphite in 300 Torr neon. The visible blackbody emission from the glowing nanoparticles was imaged after cessation of the bright plasma emission. This exposure shows three brighter particulates

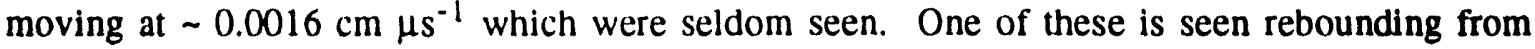
the disk used to collect the drifting nanoparticulates for analysis by Fourier Transiorm Mass Spectrometry (see alignment, lower frame). The soot collected on this disk was rich in fullerenes, while the soot deposited at early times on the graphite rod was nearly devoid of these clusters.

\section{Conclusions}

The implementation of several in situ diagnostics (optical absorption and emission spectroscopy, ion probes, and ICCD photography) has been described as a means to view the transport of ions, excited states, ground state neutrals and ions, and even hot particles and clusters in laser ablation plumes used for PLD. In order to form a comprehensive picture of the PLD environment, the diagnostics must be employed together.

Fast ICCD photography has revealed several interesting hydrodynamic effects, including: the spatial distribution of the plume in vacuum, the formation of a contact front and the coalescence of the propagating shock structure in a background gas, reflected shocks within the plume at high background gas pressures, and redeposition of material onto the target surface. The extreme low-light sensitivity of ICCD photography reveals additional effects, including: the collision of the plume with the substrate heater resulting in the formation of a stagnation layer and rebound shock, the ejection of glowing particulates from the target surface at late times and the subsequent trajectories of these particles, and the formation of hot nanoparticles following thermalization of hot plasma plumes in high pressure hackground gases. 
The propagation of PLD plumes through low-pressure background gases, as determined by ICCD photography and ion probe analysis, is best described by a combination of a drag model at early times and a generalized shock model after the coalescence of a stable 'shock' front. Ion probes are convenient and powerful tools to record the slowing and attenuation of the ion flux in the plasma plume due to scattering and collisions caused by the background gas atoms.

Gated photon counting of blackbody emission provides a useful diagnostic to determine particle temperatures and cooling rates in midflight. As in this study, this diagnostic may serve to help estimate the surface temperature of the target and resolve questions of superheating.

\section{Acknowledgments}

The author gratefully acknowledges the principal collaboration of Alex Puretzky in the graphite ablation experiments, as well as the work of R. L. Hettich, X.-Y. Zheng, R. E. Haufler, and R. N. Compton. The technical assistance of J. K. Jackson of NIST, Chris Meyers and Yair Talmi of Princeton Instruments, Inc., and G. E. Jellison, Jr., H. E. Harmon, R. Toedte, E. Arakawa, and M. M. Abraham of ORNL is also gratefully acknowledged. This publication was based upon research sponsored by the Directors R\&D Fund at Oak Ridge National Laboratory, and the Office of Health and Environmental Research and the Division of Materials Sciences, U.S. Department of Energy, under contract DE-AC05-840R21400 with Martin Marietta Energy Systems, Inc..

\section{References}

1. D. Dijkkamp, T. Venkatesan, X. D. Wu, , S. A. Shaheen, N. Jisrawi, Y. H. Min-Lee, W. L. McLean,, and M. Croft, Appl. Phys. Lett. 51, 619 (1987).

2. Laser Ablation for Matericls Synthesis, ed. by D. C. Paine and J. C. Bravman, Mat. Res. Soc. Symp. Proc., Vol. 191 (1990).

3. Laser Ablation: Mechanisms and Applications, ed. by J. C. Miller and R. F. Haglund, Springer-Verlag, Heidelberg, (1991).

4. Laser Ablation of Electronic Materials: Basic Mechanisms and Applications, ed. by E. Fogarassy and S. Lazare, North Holland (1992).

5. Laser Ablation in Materials Processing: Fundamentals and Applications, ed. by B. Braren, J.J. Dubowski, and D. P. Norton, Mat. Res. Soc. Symp. Proc., Vol. 285 (1993).

6. Laser Ablation II: Mechanisms and Applications, ed. by J. C. Miller and D.B. Geohegan, AIP Conference Proceedings 288, (1993).

7. Pulied Laser Deposition of Thin Films, ed. by D. B. Chrisey and G. K. Hubler, (WileyInterscience Publisher), 1993, (in press).

8. J. T. Cheung, and H. Sankur, , CRC Critical Review in Solid State and Materials Sciences 15, 63 (1988) and references cited therein.

9. J. F. Ready, Effects of High-Power Laser Radiation, Academic, London (1971).

10. T. P. Hughes, Plasmas and Laser Light, Wiley, New York (1975).

11. G. Bekefi, Principles of Laser Plasmas, New York, (1976).

12. L. J. Radziemski, D. A. Cremers, Laser Induced Plasmas and Applications, Marcel Dekker, Inc., New York, (1989).

13. See review by D. B. Geohegan, chapter in Reference 7.

14. D. B. Geohegan and D. N. Mashburn, p. 211 in Reference 2.

15. D. B. Geohegan, p. 28 in Reference 3.

16. D. B. Geohegan, pp. 73-88 in Reference 4.

17. D. B. Geohegan, p. 27 in Reference 5.

18. D. B. Geohegan, in Reference 6 (in press). 
19. D. B. Geohegan, p. 557 in Surface Chemistry and Beam-Solid Interactions, Mat. Res. Soc. Symp. Proc. 201 (1991).

20. D. B. Geohegan, Appl. Phys. Lett. 62, 1463 (1993).

21. D. B. Geohegan, Thin Solid Films 220, 138 (1992).

22. D. B. Geohegan, Appl. Phys. Lett., 60, 2732 (1992).

23. D. B. Geohegan and D. N. Mashburn, Appl. Phys. Lett. 55, 2345 (1989).

24. A. D. Akhsakhalyan, Yu. A. Bityurin, S. V. Gaponov, A. A. Gudkov, and V. I. Luchin, Sov. Phys. Tech. Phys., 27, 969, (1982).

25. Ya. B. Zel'dovich and Yu. P. Raizer, p. 94. in Physics of Shock Waves and High Temperature Hydrodynamic Phenomena, Vol. 1, Academic Press, New York, 1966.

26. P. E. Dyer, A. Issa,, and P. H. Key, Appl. Phys. Lett. 57, 186 (1990).

27. P. E. Dyer, A. Issa, and P. H. Key, Appl. Surf. Sci. 46, 89 (1990).

28. R. M. Gilgenbach and P. L. G. Ventzek. Appl. Phys. Lett. 58, 1597 (1991).

29. P. E. Dyer, S. R. Farrar, A. Issa, and P. H. Key, p.101 in Reference 4.

30. P. E. Dyer, and J. Sidhu, J. Appl. Plyys. 64, 4657 (1988).

31. G. Kubiak in Reference 6.

32. H. Dupendant, J. P. Gavigan, D. Givord, A. Lienard, J. P. Rebouillat, and Y. Souche, Appl. Surf. Sci. 43, 369 (1989).

33. K. Murakami, p.125 in Reference 4.

34. A. A. Ivanov, S. G. Galkin, A. V. Kuznetsov, A. P. Menushenkov, Physica $C$ 180, 69 (1991).

35. G. Koren, A. Gupta, R. J. Baseman, M. I. Lutwyche, and R. B. Laibowitz, Appl. Phys. Lett. 56, 2144 (1990).

36. Deepika Bhattacharya, R. K. Singh, and P. H. Holloway, J. Appl. Phys. 70, 5433 (1991).

37. R. Kelly, J. J. Cuomo, P. A. Leary, J. E. Rothenberg, B. E. Braren and C. F. Aliotta, Nucl. Instrum. Methods B 9, 329 (1985).

38. R. K. Singh, D. Bhattacharya, and J. Narayan, Appl. Phys. Lett. 57, 2022 (1990).

39. Eric A. Rohlfing, J. Chem. Phys. 89, 6103 (1988).

40. H. W. Kroto, J. R. Heath, S. C. O'Brien, R. F. Curl, and R. E. Smalley, Nature 318, 162 (1985).

41. E. A. Rohlfing, D. M. Cox, A. Kaldor, J. Chem. Phys. 81, 3322 (1984).

42. R. E. Haufler, Y. Chai, L. P. F. Chibante, J. Conceicao, C. Jin, L.-S. Wang, S. Maruyama, R. E. Smalley, Mater. Res. Soc. Symp. Proc. 206, 627 (1990).

43. Y. Chai, T. Guo, C. Jin, R. E. Haufler, L.P.F. Chibante, J. Fure, L. Wang, J. M. Alford, R. E. Smalley, J. Phys. Chem. 95, 7564 (1991).

44. T. Guo, C. Jin, R. E. Smalley, J. Phys. Chem. 95, 4948 (1991).

45. A. A. Puretzky, D. B. Geohegan, R. E. Haufler, R. L. Hettich, X.-Y. Zheng, and R. N. Compton, in Reference 6.

46. D. B. Geohegan, A. A. Puretzky, R. L. Hettich, X.-Y. Zheng, R. E. Haufler, and R. N. Compton, accepted for the proceedings of the IUMRS-ICAM Conference,Tokyo, Japan. (Aug. 31-Sept. 4, 1993) (in press). 


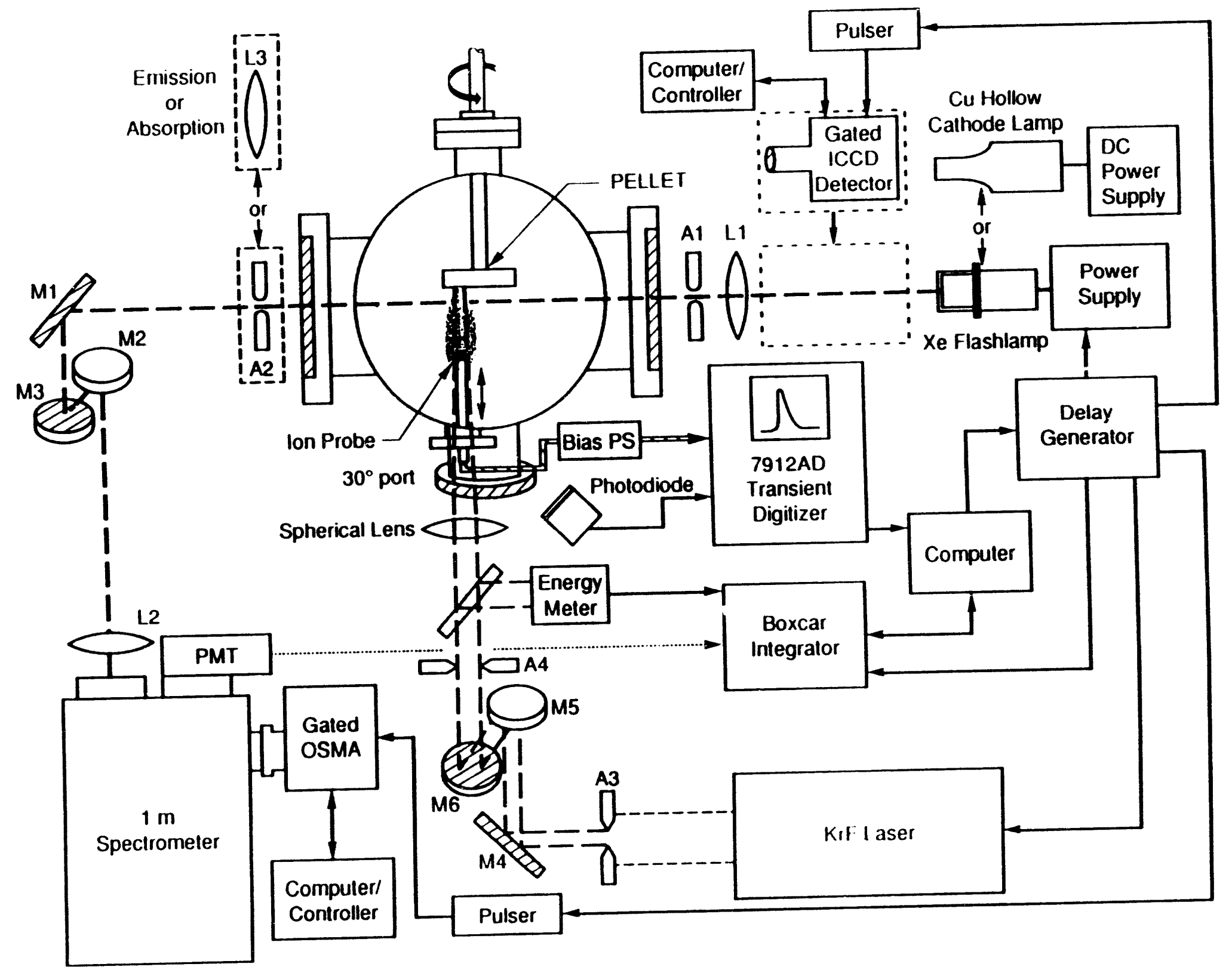



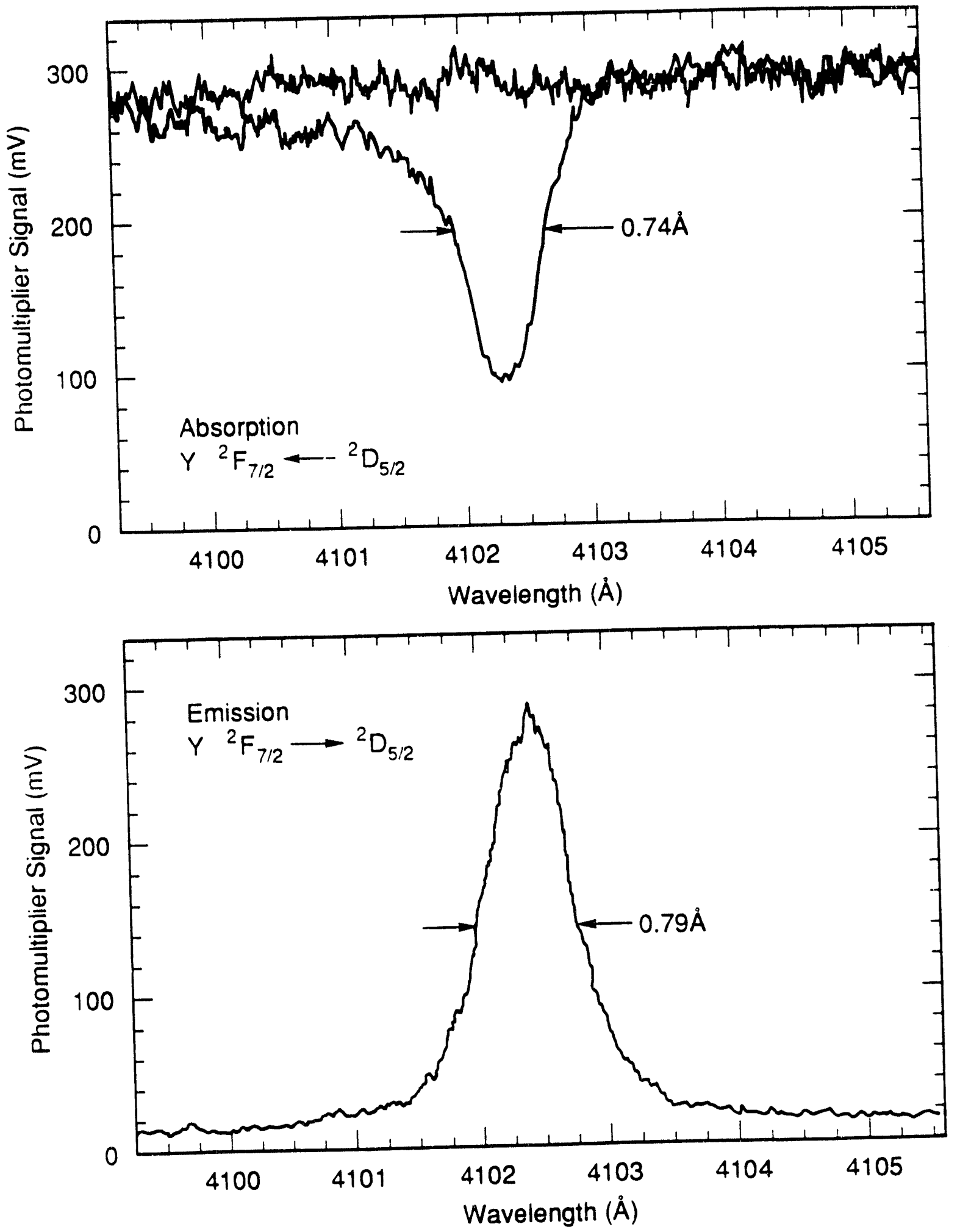

Geohegan

NATO Figure 2 

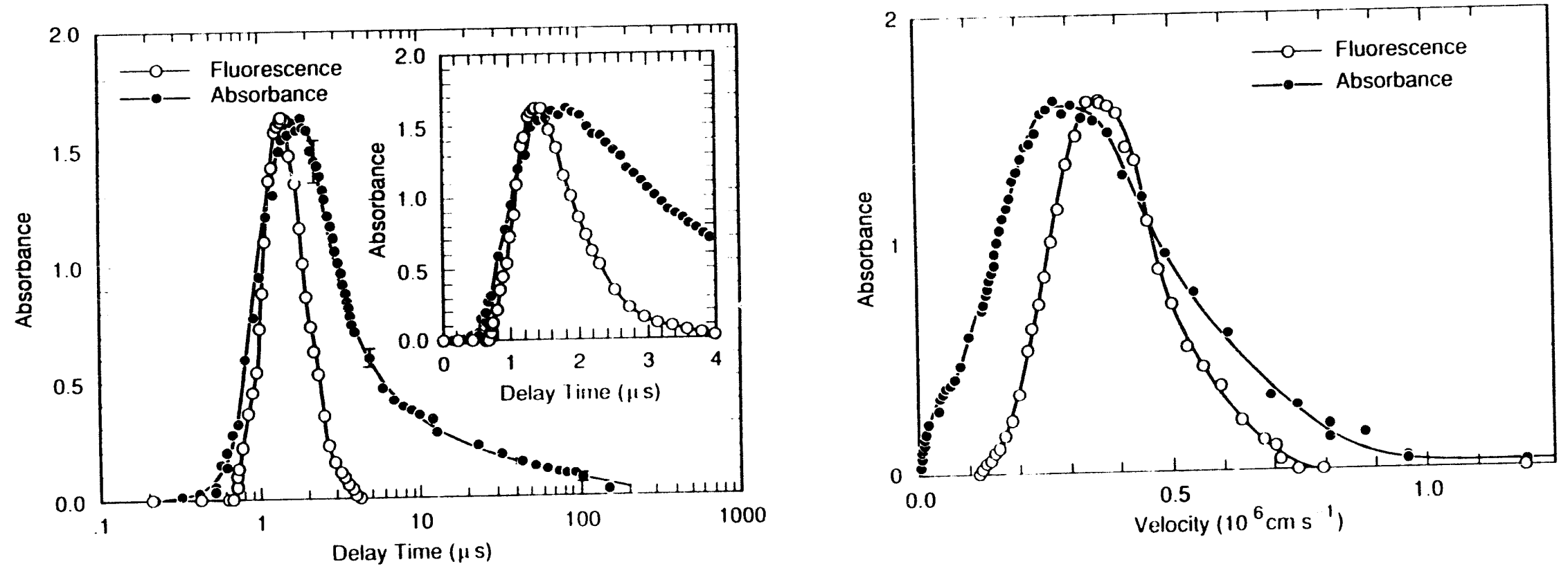

Geohegan

NATO Figure 3 

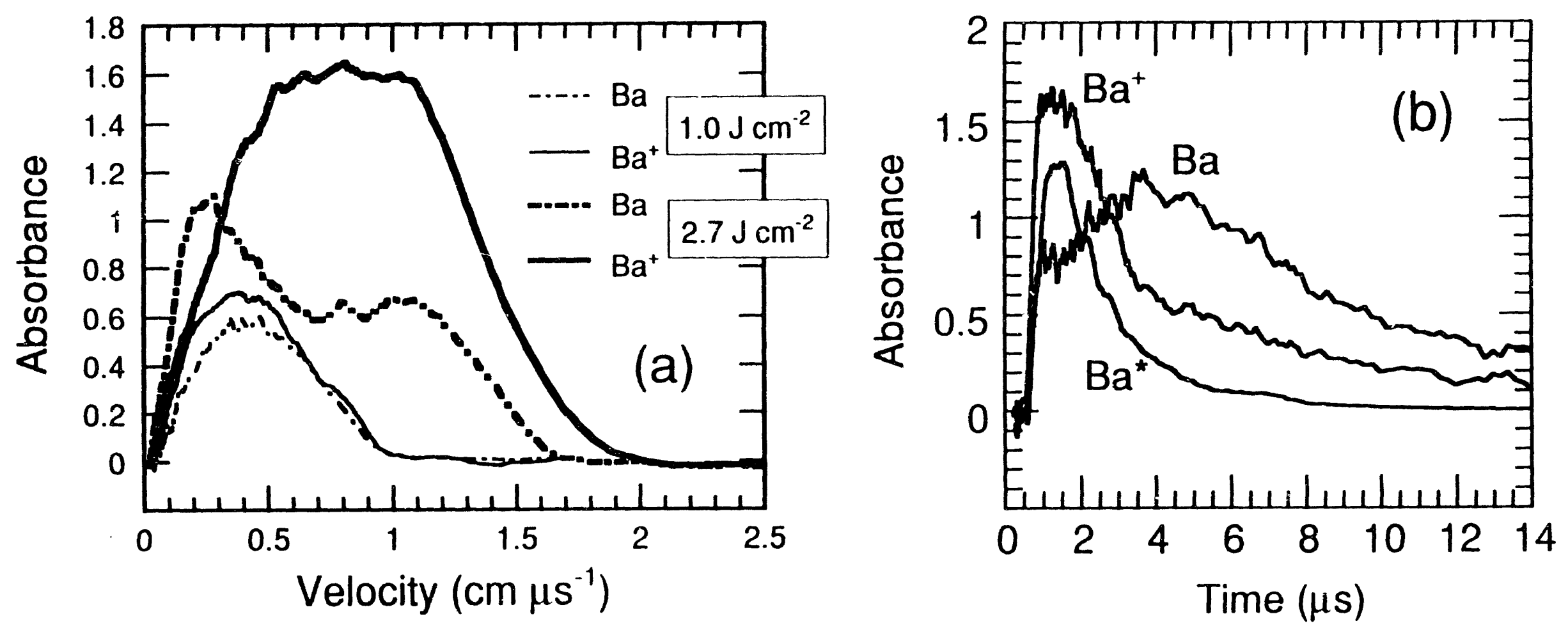

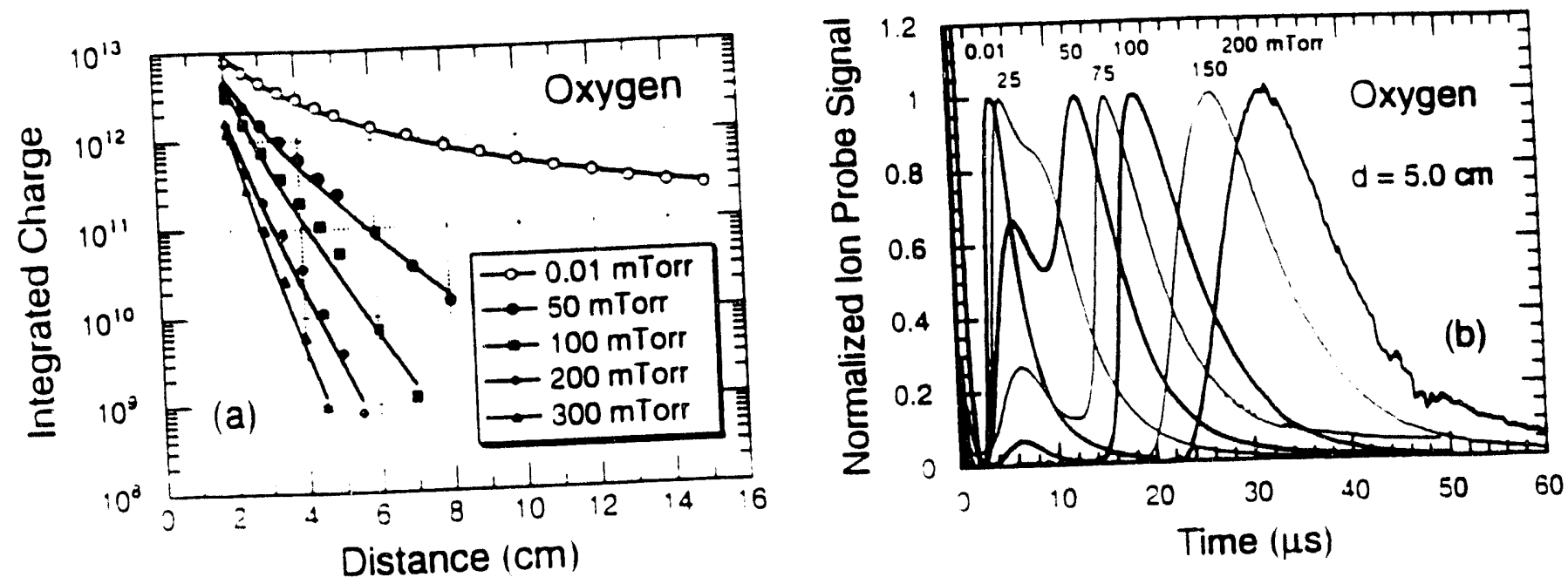


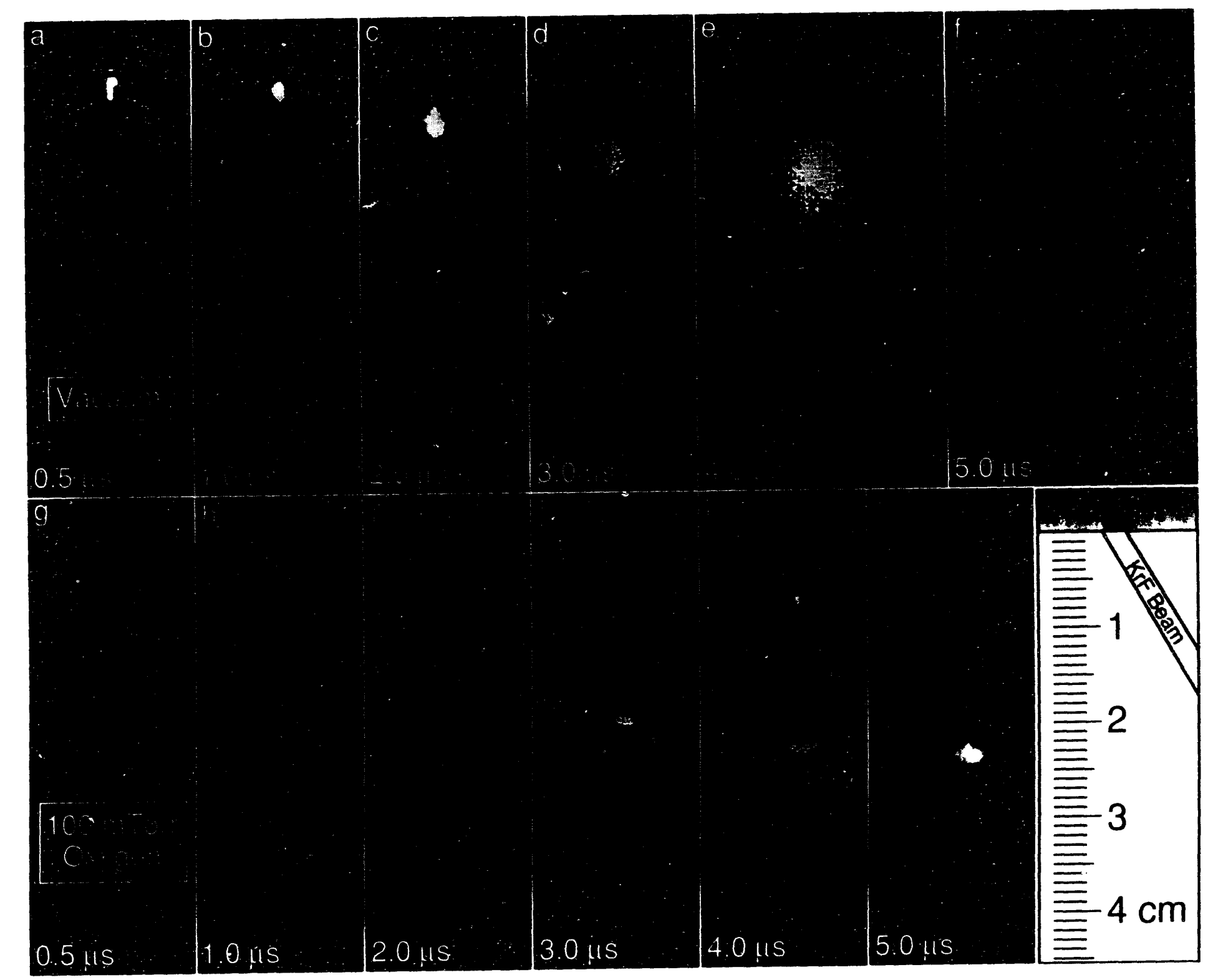

Geohegan

NATO Figure 6 


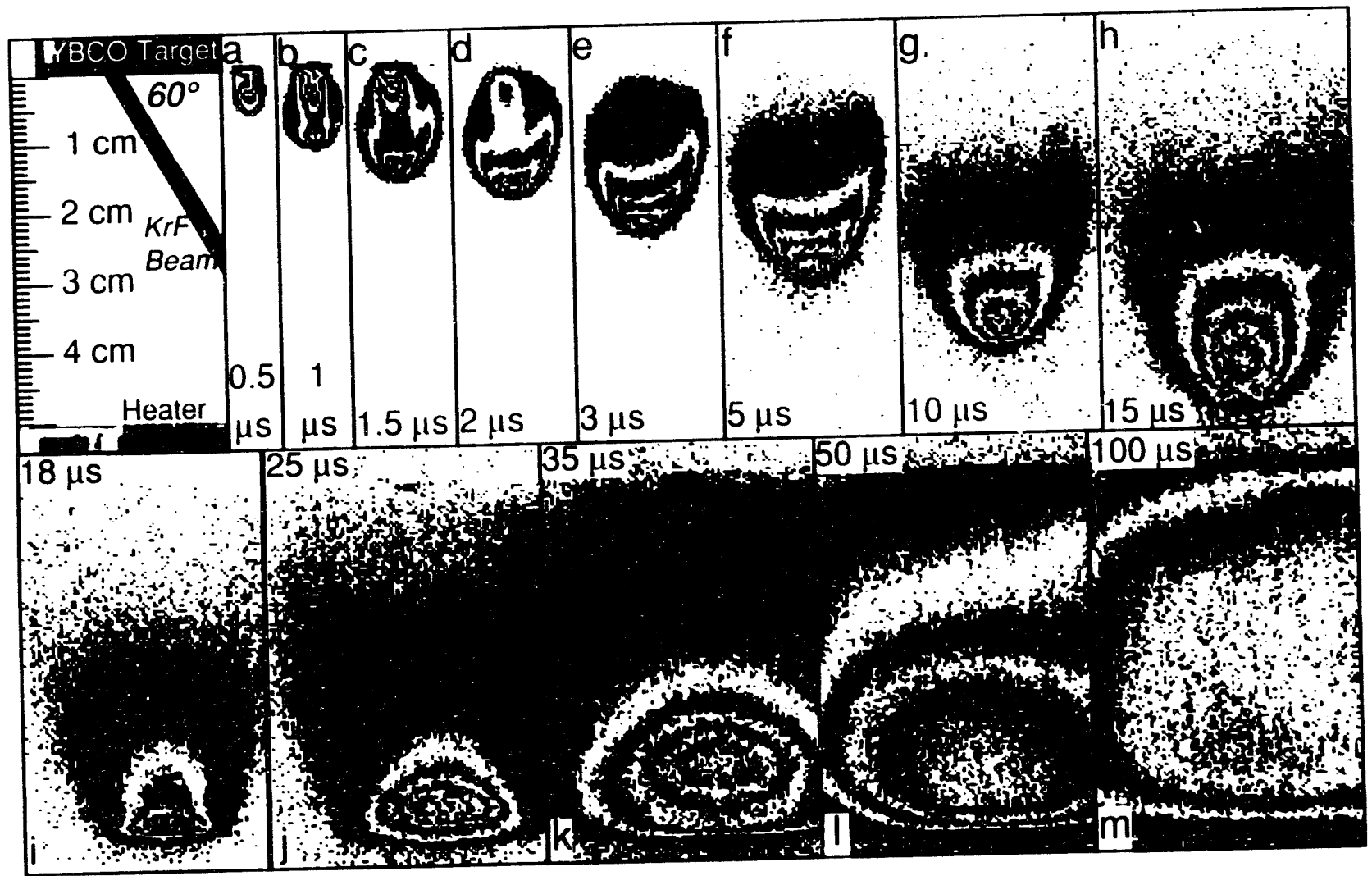

Geohegan

NATO Figure 7 

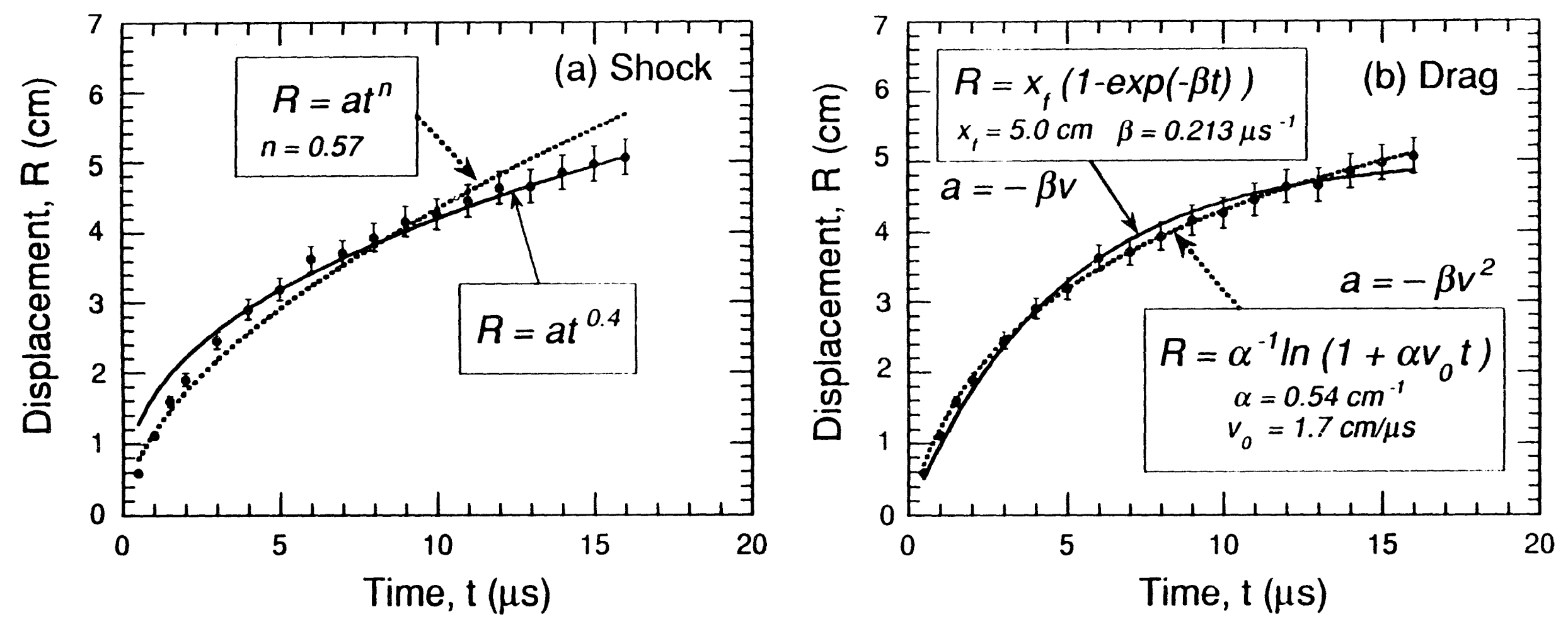

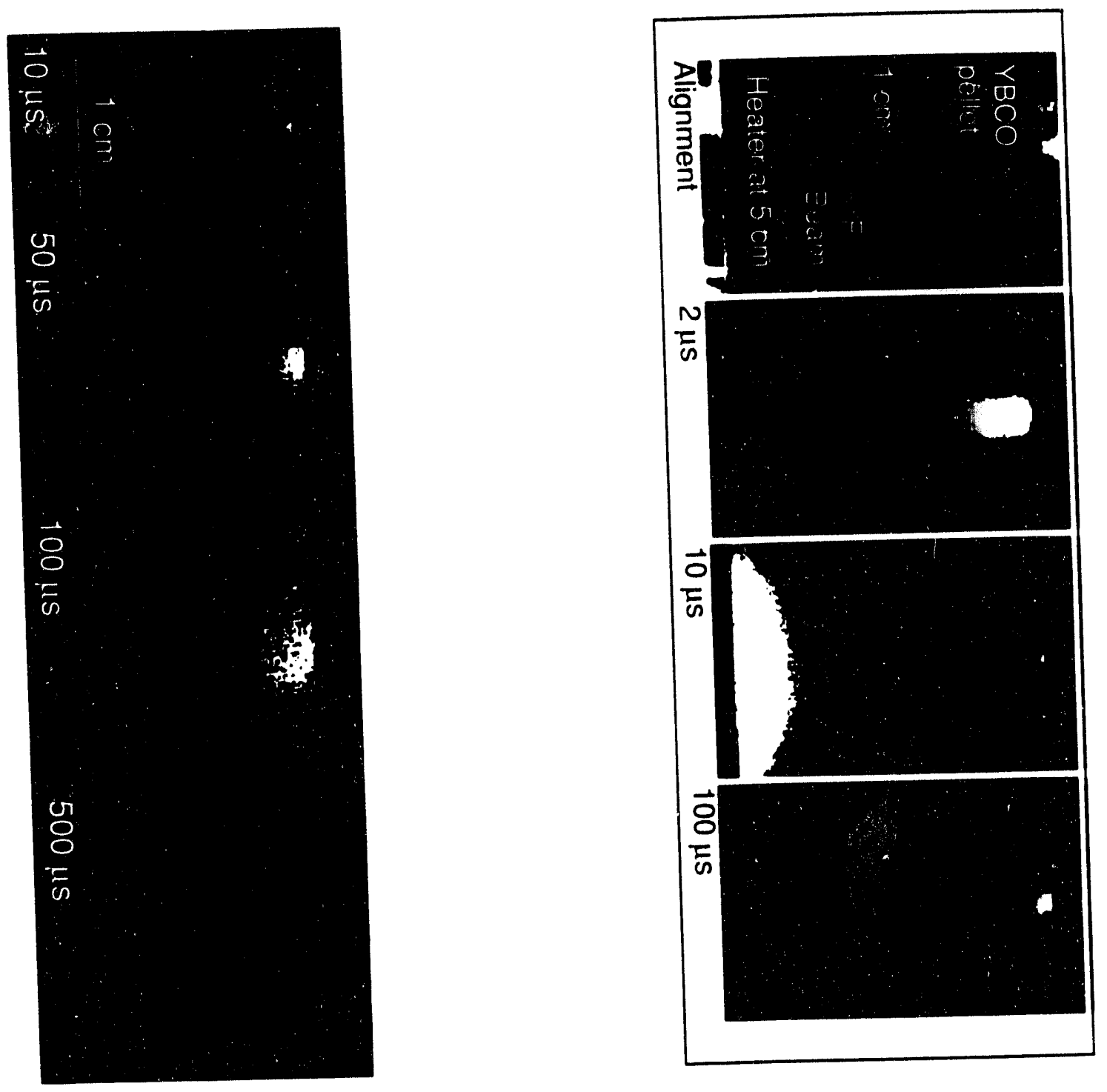

Geohegan

NATO Figure 10
Geohegan

NATO Figure 9 

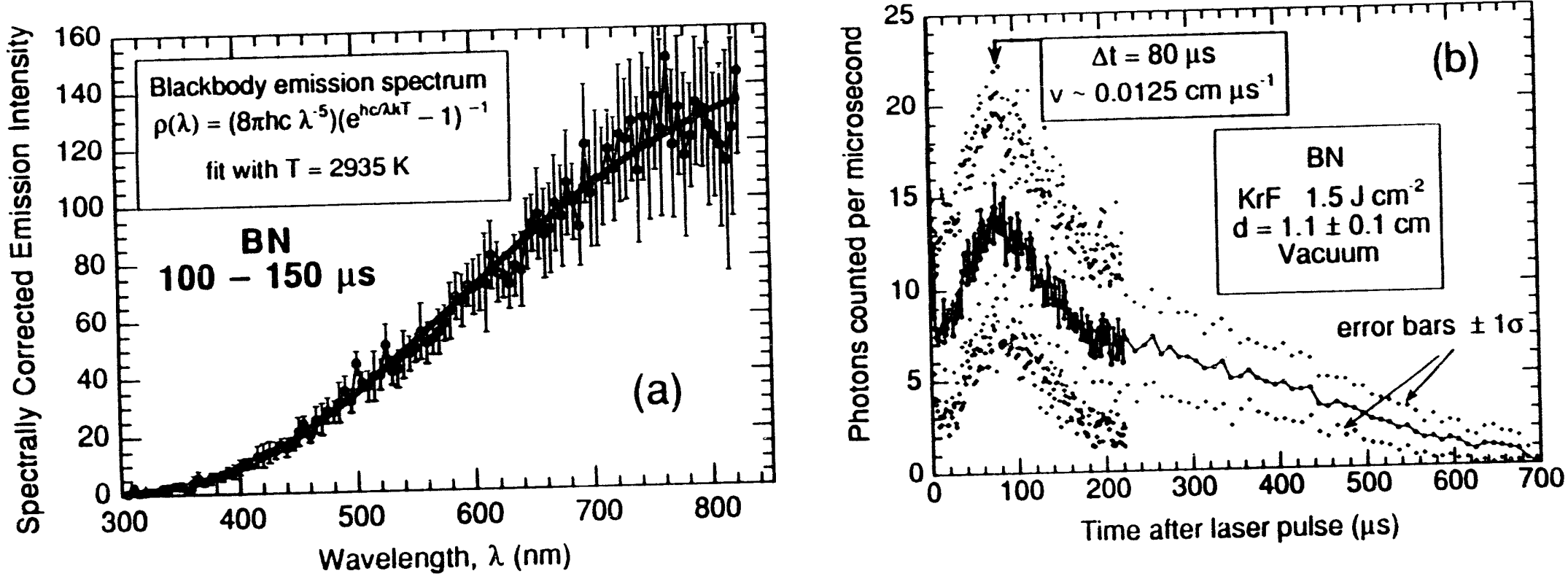

Geohegan

NATO Figure 11 

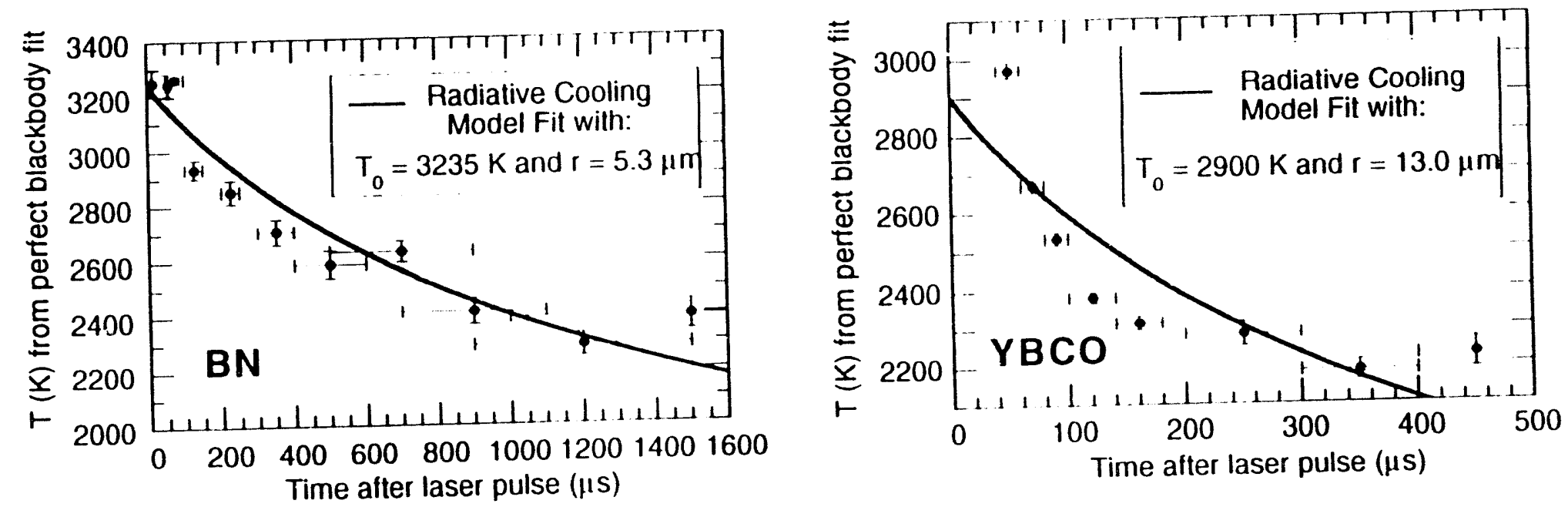

Geohegan

NATO Figure 12 


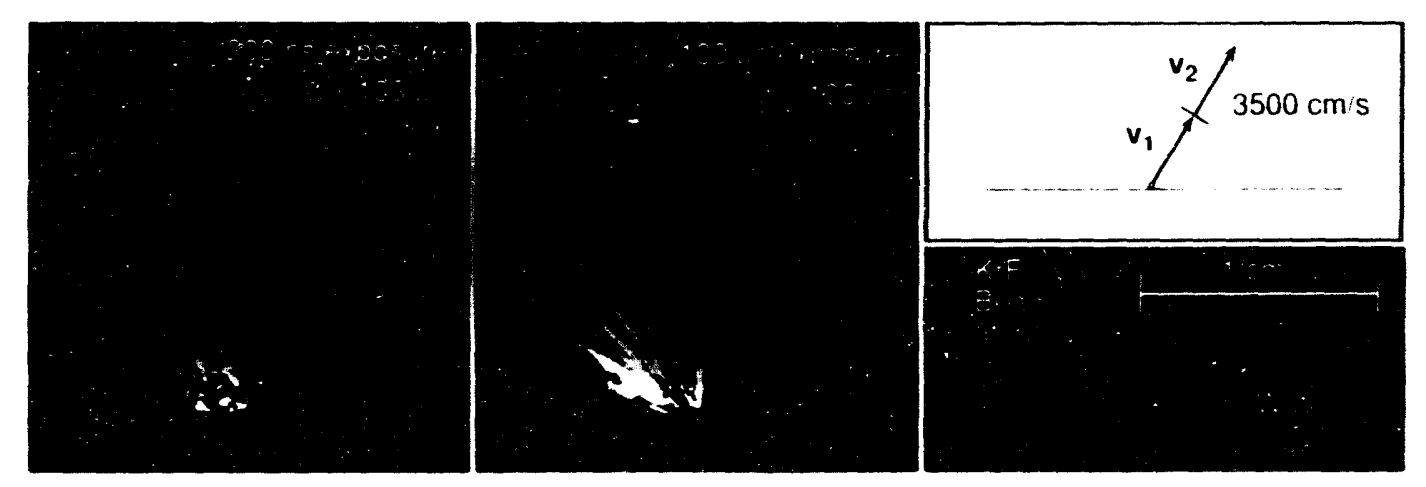

Geohegan

NATO Figure 13 


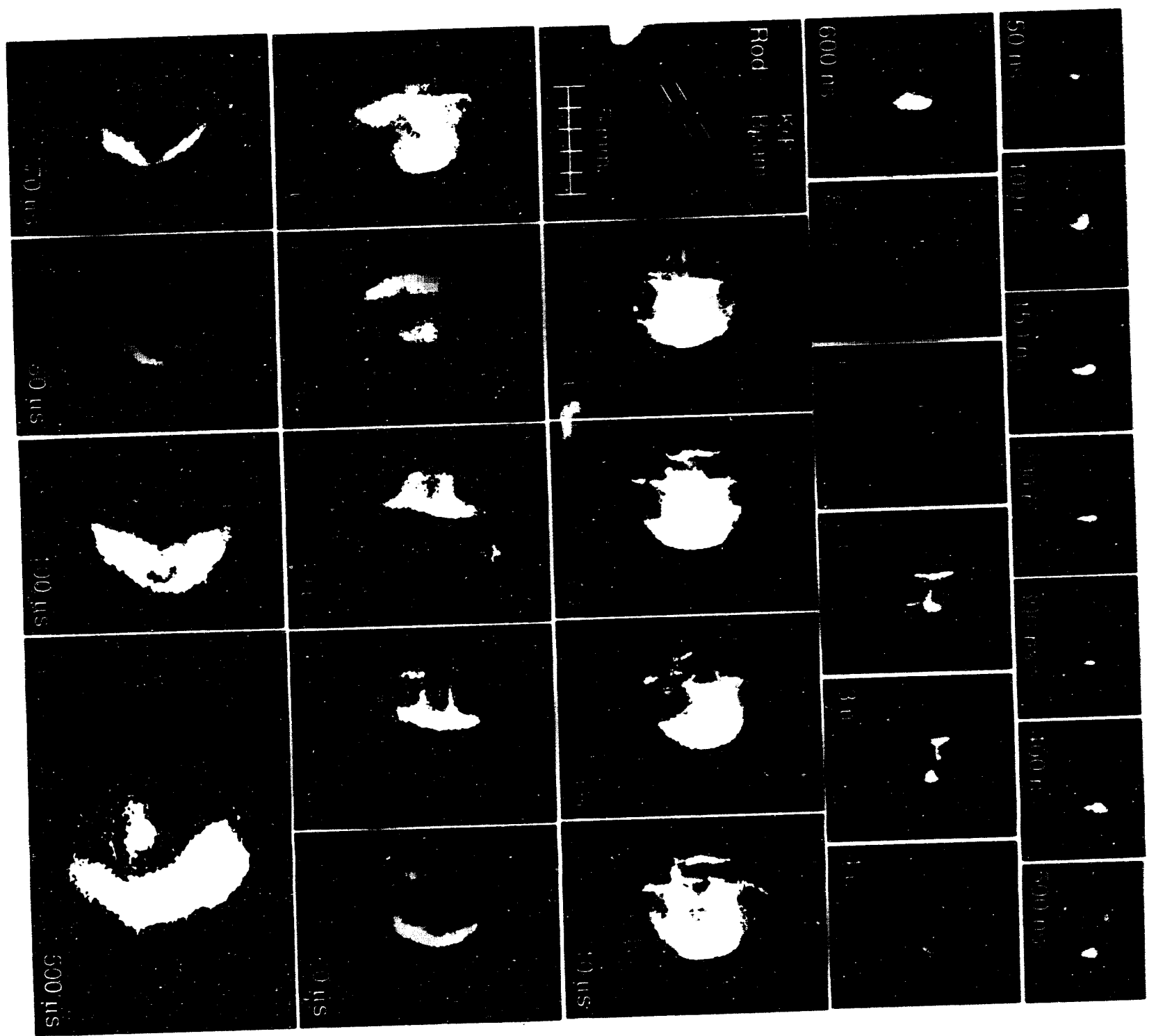




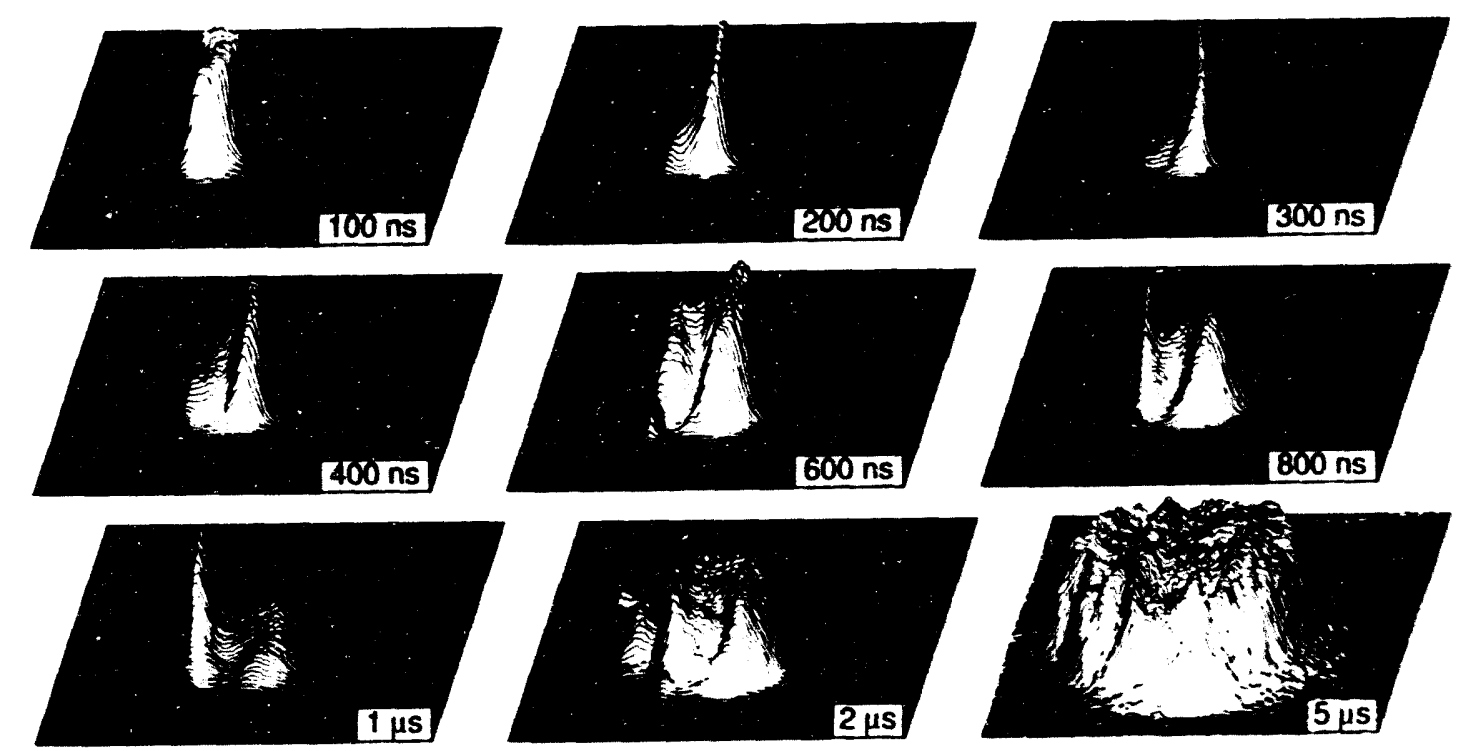

Geohegan

NATO Figure 15 


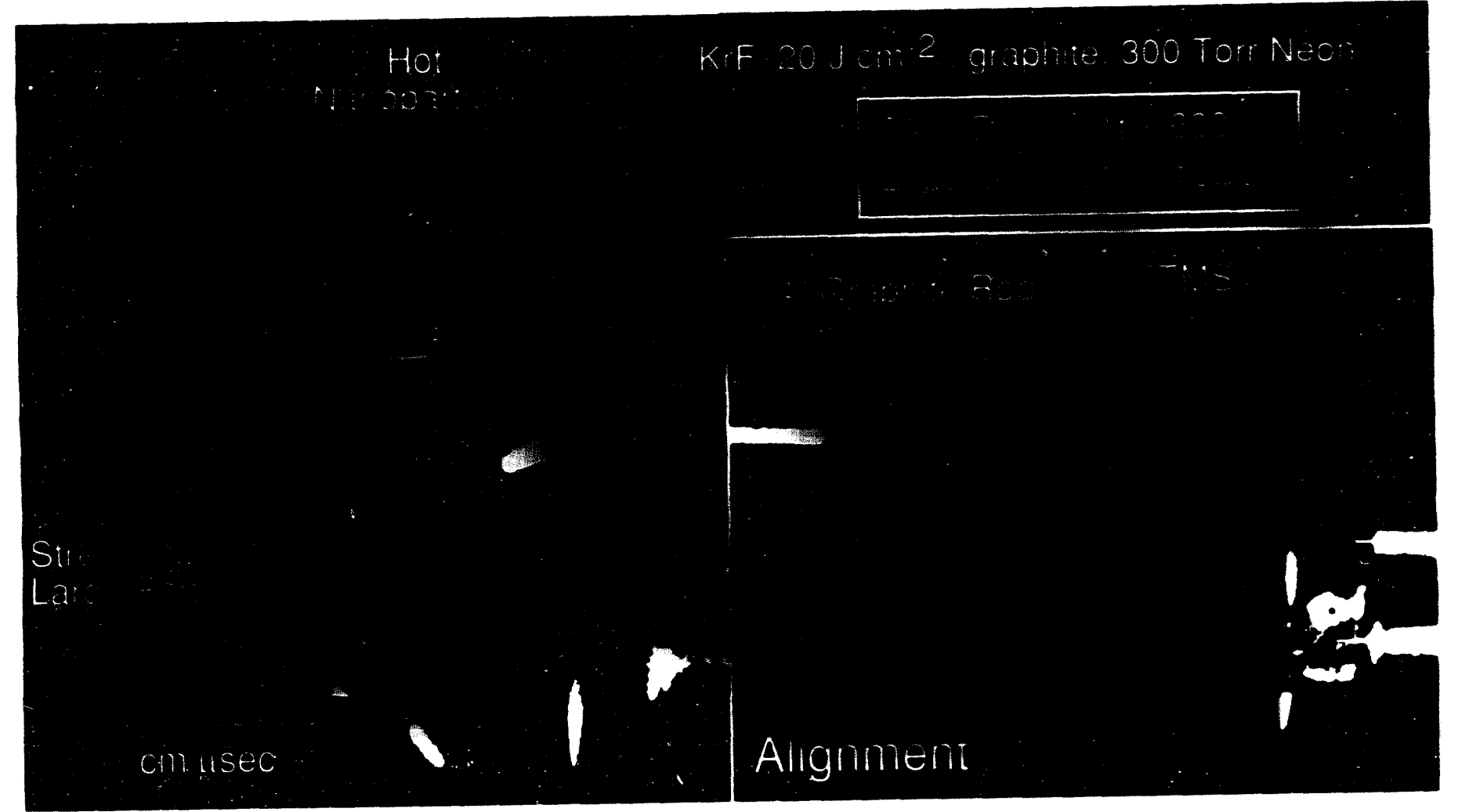

Geohegan

NATO Figure 16 

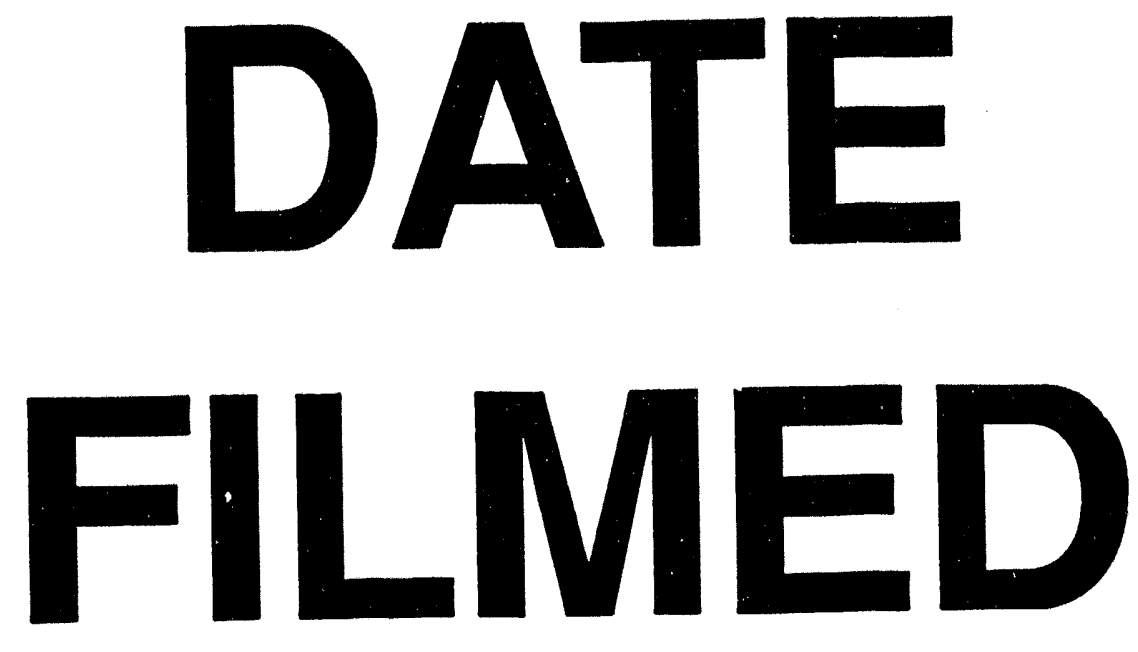

$10 / 28 / 94$
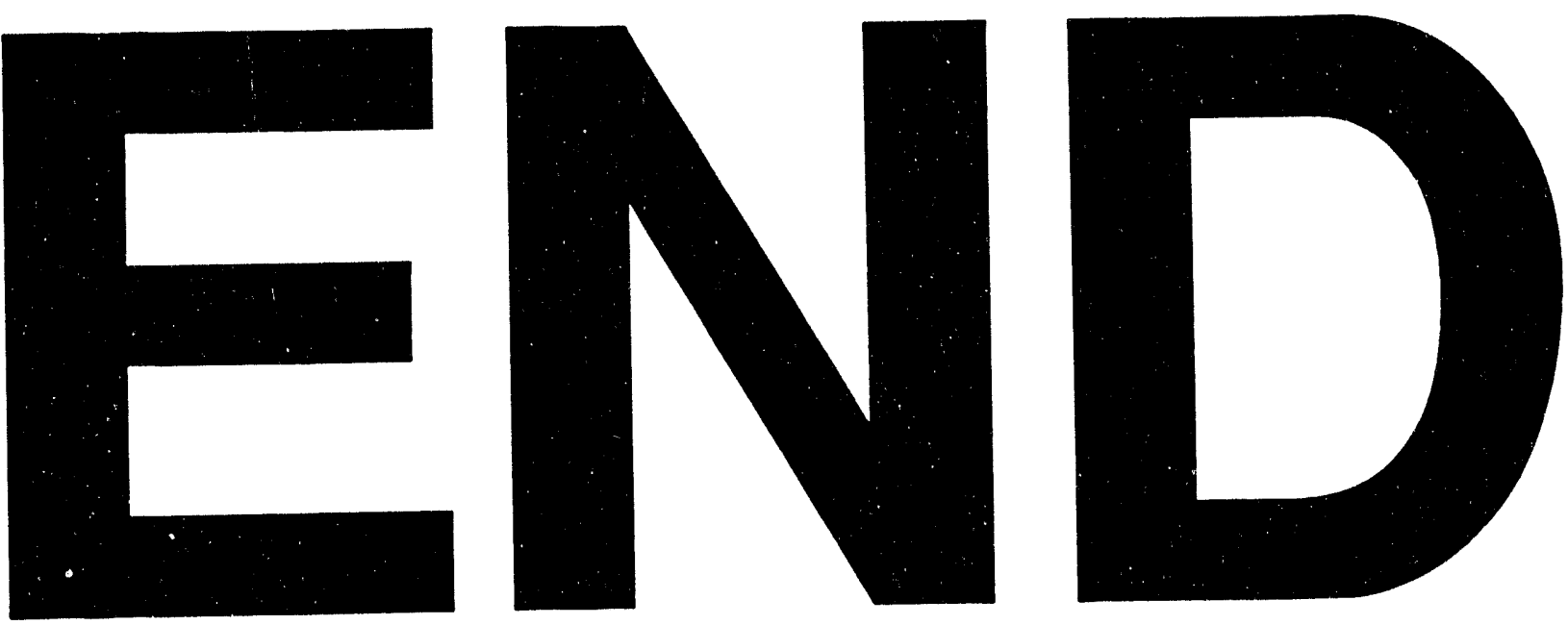

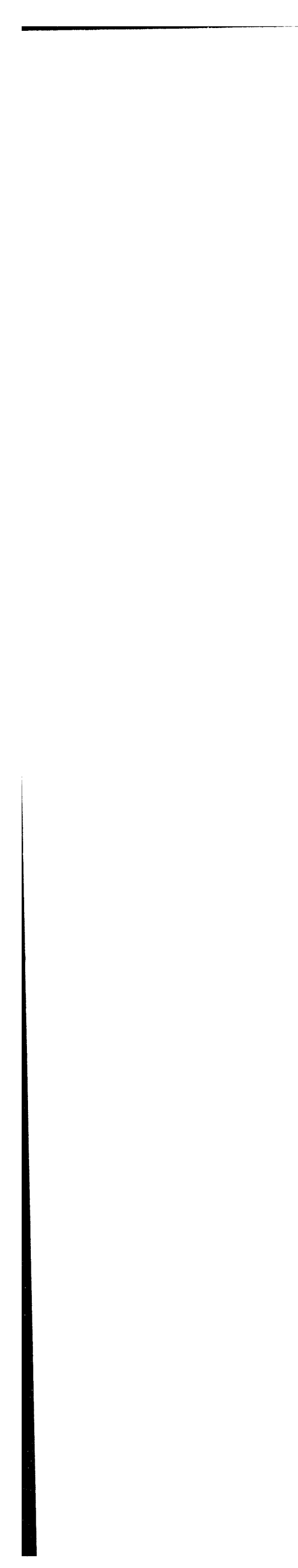

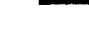

